\title{
Multidimensional Tuning in Motor Cortical Neurons during Active Behavior
}

\author{
Rachel C. Yuan ${ }^{1}$ and ${ }^{D S}$ Sarah W. Bottjer ${ }^{2}$
}

https://doi.org/10.1523/ENEURO.0109-20.2020

${ }^{1}$ Neuroscience Graduate Program, University of Southern California, Los Angeles, CA 90089 and ${ }^{2}$ Section of Neurobiology, University of Southern California, Los Angeles, CA 90089

\begin{abstract}
A region within songbird cortex, dorsal intermediate arcopallium (Ald), is functionally analogous to motor cortex in mammals and has been implicated in song learning during development. Non-vocal factors such as visual and social cues are known to mediate song learning and performance, yet previous chronic-recording studies of regions important for song behavior have focused exclusively on neural activity in relation to song production. Thus, we have little understanding of the range of non-vocal information that single neurons may encode. We made chronic recordings in Ald of freely behaving juvenile zebra finches and evaluated neural activity during diverse motor behaviors throughout entire recording sessions, including song production as well as hopping, pecking, preening, fluff-ups, beak interactions, scratching, and stretching. These movements are part of natural behavioral repertoires and are important components of both song learning and courtship behavior. A large population of Ald neurons showed significant modulation of activity during singing. In addition, single neurons demonstrated heterogeneous response patterns during multiple movements (including excitation during one movement type and suppression during another), and some neurons showed differential activity depending on the context in which movements occurred. Moreover, we found evidence of neurons that did not respond during discrete movements but were nonetheless modulated during active behavioral states compared with quiescence. Our results suggest that Ald neurons process both vocal and non-vocal information, highlighting the importance of considering the variety of multimodal factors that can contribute to vocal motor learning during development.
\end{abstract}

Key words: motor cortex; multimodal; sensorimotor; songbird

\section{Significance Statement}

Motor cortex across taxa receives highly integrated, multimodal information and has been implicated in both execution and acquisition of complex motor skills, yet studies of motor cortex typically employ restricted behavioral paradigms that target select movement parameters, preventing wider assessment of the diverse sensorimotor factors that can affect motor cortical activity. Recording in dorsal intermediate arcopallium (Ald) of freely behaving juvenile songbirds that are actively engaged in sensorimotor learning offers unique advantages for elucidating the functional role of motor cortical neurons. The results demonstrate that a diverse array of factors modulate motor cortical activity and lay important groundwork for future investigations of how multimodal information is integrated in motor cortical regions to contribute to learning and execution of complex motor skills. 


\section{Introduction}

Goal-directed skill learning underlies our ability to acquire motor skills and flexibly perform them in response to changing environmental contexts. Both learning and performance of motor skills are highly sensorimotor processes: successful acquisition of motor behaviors entails integration across internal and external sources of sensory feedback to guide accurate refinement of motor output. Correspondingly, motor cortical neurons demonstrate multidimensional tuning that reflects integration across a variety of inputs: motor cortical neurons involved in performance of skilled behaviors have been shown to encode not only motor parameters (e.g., movement force or direction) but also non-motor parameters such as preparatory activity before movement execution or visual information specific to a target object's location in object-directed reaching tasks (Tanji and Evarts, 1976; Evarts and Fromm, 1977; Murata et al., 1997; Shen and Alexander, 1997; Ferezou et al., 2007). In addition, increasing evidence indicates that motor cortex serves not only as a driver of learned movements but also as a central locus for the acquisition of new motor skills across a variety of movements and training paradigms (Whishaw et al., 1991; Whishaw, 2000; Darling et al., 2011; Guo et al., 2015; Kawai et al., 2015; Makino et al., 2016; Peters et al., 2017; Papale and Hooks, 2018; Hwang et al., 2019). These findings suggest motor cortex as a dynamic substrate that actively integrates diverse streams of information to contribute to sensorimotor learning and performance. However, the potential influence of various multimodal inputs on motor cortical activity during behavior is difficult to assess in anesthetized and/or restrained experimental paradigms that focus on a single motor task; recordings in freely behaving animals, especially in the context of sensorimotor skill learning, afford opportunities for investigating sensorimotor integration in motor cortical neurons (Ebbesen et al., 2017; Zhang et al., 2017; Mimica et al., 2018).

Vocal learning in juvenile songbirds entails integration of social cues as well as visual, auditory, and somatosensory information to guide refinement of variable babbling into stereotyped song (Price, 1979; West and King, 1988; Eales, 1989; Mann et al., 1991; Mann and Slater, 1995; King et al., 2005; Derégnaucourt et al., 2013; Chen et al., 2016; Ljubičić et al., 2016; Carouso-Peck and Goldstein, 2019). Moreover, song production occurs in the context of other social behaviors such as hopping, beak interactions, and preening. For example, adult males must combine both vocal and non-vocal elements in an integrated performance to successfully court females (Morris, 1954; Williams, 2001; Cooper and Goller, 2004; Tomaszycki and

This work was supported by National Institute of Neurological Disorders and Stroke Grant F31NS092299 and R56NS098744 and by the National Institute on Deafness and Other Communication Disorders Grant T32DC009975.

Acknowledgements: We thank Christopher Meyer for technical assistance.

Correspondence should be addressed to Rachel C. Yuan at rachelyu@ usc.edu.

https://doi.org/10.1523/ENEURO.0109-20.2020

Copyright $@ 2020$ Yuan and Bottjer

This is an open-access article distributed under the terms of the Creative Commons Attribution 4.0 International license, which permits unrestricted use, distribution and reproduction in any medium provided that the original work is properly attributed.
Adkins-Regan, 2005; Dalziell et al., 2013; Ota et al., 2015; Ullrich et al., 2016). Thus, songbirds offer a model system for examining multiple diverse behaviors during acquisition and performance of a complex motor skill. However, despite evidence for the importance of nonvocal factors in song learning and performance, few studies of regions that process song-related information have examined neural activity in relation to nonvocal behaviors.

In songbirds, the dorsal intermediate arcopallium (Ald) has been considered to be analogous to motor cortex in mammals and has been implicated in both vocal learning and non-vocal motor behavior (Feenders et al., 2008; Bottjer and Altenau, 2010; Karten, 2013). Ald receives inputs that process multimodal sensory information via dorsal caudolateral nidopallium (dNCL) as well as information from cortico-basal ganglia circuitry that mediates vocal learning (via LMAN-sHELL) and, in turn, makes a variety of projections that give rise to feedforward and feedback pathways through subcortical and brainstem regions (Fig. 1A; Zeier and Karten, 1971; Bottjer et al., 2000; Paterson and Bottjer, 2017). Ald is thus well suited to integrate multiple sources of external and internal sensory information to contribute to motor skill learning and performance.

We made chronic recordings in Ald of freely behaving juvenile songbirds during the sensorimotor period of vocal learning and analyzed the activity of single neurons during singing as well as during several discrete behaviors performed as part of the natural repertoire of zebra finches. This novel approach enabled us to explore the idea that neural activity patterns in brain regions that mediate vocal learning are not restricted to song production. Our results represent an extensive assessment of motor cortical activity across a wide variety of natural behaviors, thereby informing our understanding of how these neurons may contribute to motor skill learning and production.

\section{Materials and Methods}

\section{Subjects}

All animal procedures were performed in accordance with the University of Southern California animal care committee's regulations. Seven male juvenile zebra finches [43-58 days post-hatch (dph); mean age $46 \mathrm{dph}$ on first day of recording] were used; this age range corresponds to the early stages of the sensorimotor learning period, when birds have completed memorization of vocal sounds from social tutors and are producing immature, variable vocalizations. Female zebra finches do not produce song and were therefore excluded from this study. All birds were raised in group aviaries until at least $33 \mathrm{dph}$, remaining with their natural parents and thereby receiving normal auditory and social experience during the tutor memorization period (Immelmann, 1969; Böhner, 1983, 1990; Eales, 1985; Mann and Slater, 1995; Roper and Zann, 2006). Juveniles were separated from group aviaries at 33-35 dph and housed in single cages within the experimental rig. Each bird's tutor was placed in a separate cage within view of the juvenile to help it acclimate to 
A

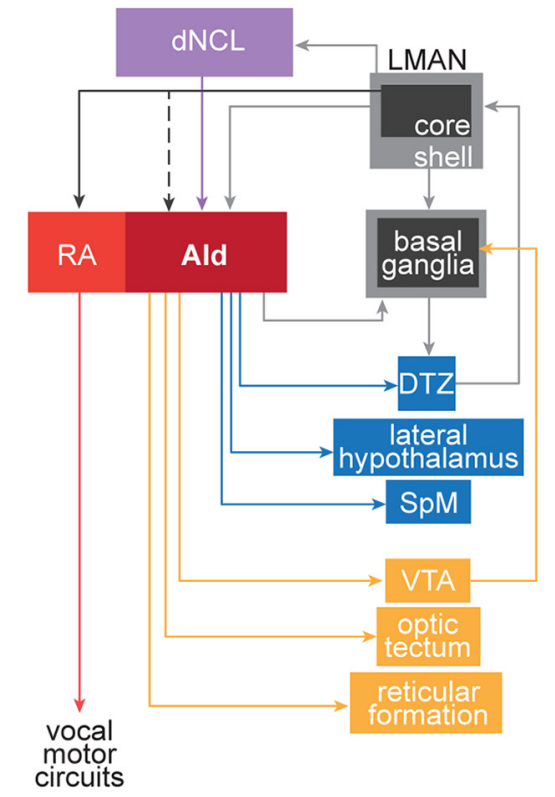

C
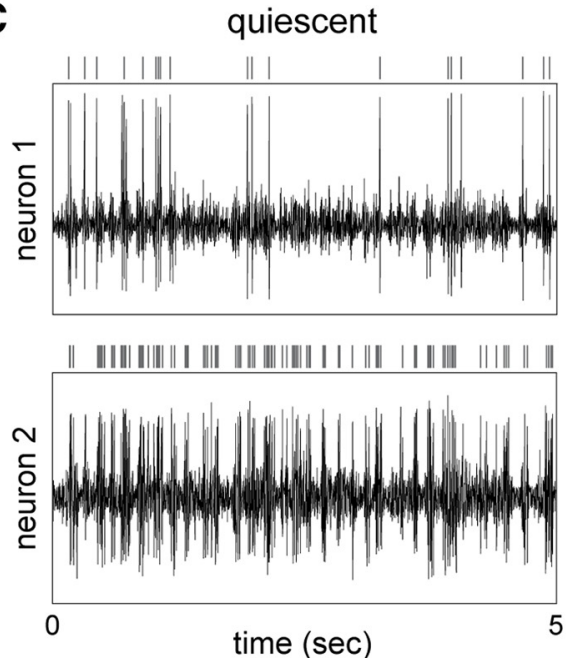

B
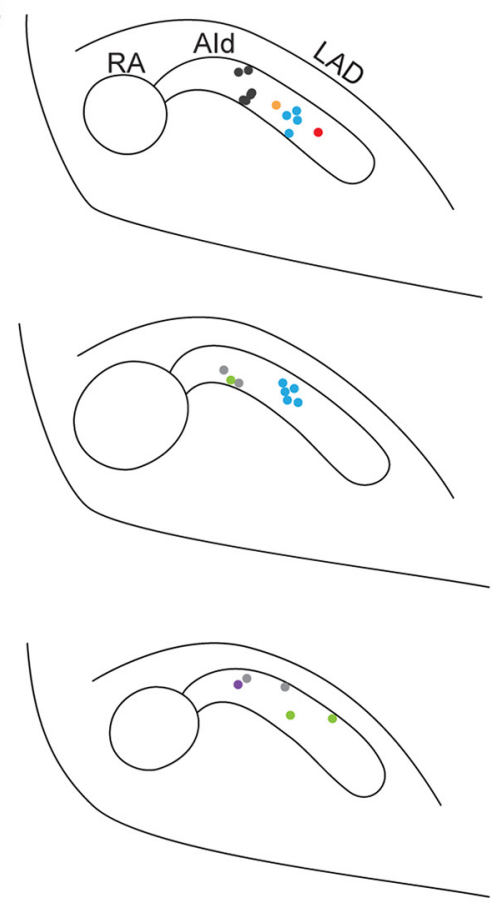

actively behaving

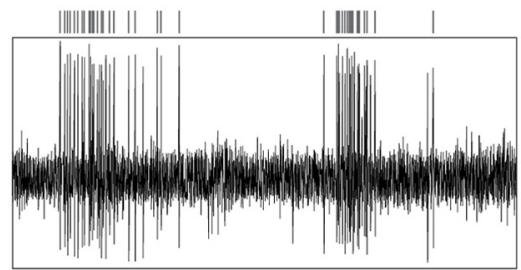

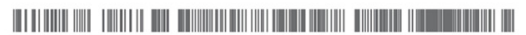

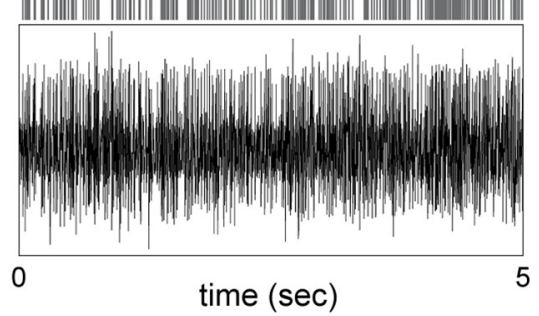

Figure 1. Ald neurons are well situated to integrate multimodal inputs and distribute information across various cortical-subcortical circuits. $\boldsymbol{A}$, Ald receives inputs from upstream cortical regions LMAN-SHELL and dNCL. LMAN-SHELL is part of a cortico-basal ganglia loop that mediates vocal learning, whereas dNCL receives inputs from LMAN-SHELL as well as multiple pathways processing somatosensory, visual, and auditory information. Ald of juvenile birds also receives inputs from LMAN-CORE via axon collaterals of LMAN-CORE $\rightarrow$ RA neurons that drive vocal output; robust collaterals are present in juvenile birds before $\sim 40-45 \mathrm{dph}$ but are not present in older juvenile or adult birds. Projections from Ald to striatum and several midbrain and thalamic regions give rise to both feedback and feed-forward pathways, creating several opportunities for information transfer between cortical and subcortical regions. DTZ, dorsal thalamic zone (includes both DLM and DMP); LMAN, lateral magnocellular nucleus of the anterior nidopallium; RA, robust nucleus of the arcopallium. $\boldsymbol{B}$, top to bottom, Caudal-to-rostral series of coronal sections $250 \mu \mathrm{m}$ apart mapping locations of recordings made in Ald. Different colored circles represent sites recorded from different birds $(n=7)$. $\boldsymbol{C}$, Raw traces of extracellular activity simultaneously recorded at two different sites within Ald of a juvenile bird (44 dph) while the bird was resting (left column; "quiescent") versus hopping around the recording cage (right column; "actively behaving"). Vertical lines above each raw activity trace indicate spikes from a single neuron sorted from the extracellular activity. 
the experimental rig for $2-5 d$ before the start of recording.

\section{Anatomy}

We refer to our region of interest as Ald, following the terminology of Reiner et al. (2004). Ald extends laterally from the vocal motor nucleus RA (robust nucleus of the arcopallium) and is coterminous with RA. Ald receives direct input from both LMAN-SHELL and dNCL and projects to several downstream targets, including the ventral tegmental area (VTA; Fig. 1A; Bottjer et al., 2000). Many sources of afferent input converge in the intermediate arcopallium and different arcopallial subregions in turn make a variety of downstream projections, resulting in a complex and heterogeneous area. It seems possible that Ald as defined by afferent inputs from LMAN-SHELL may overlap slightly with AIV, which was defined by Mandelblat-Cerf et al. (2014) as an area of intermediate arcopallium that receives inputs from higher-level auditory cortical regions and projects to VTA (Gale et al., 2008). In addition, AIV clearly overlaps with or is identical to a region known as "RA cup," which is a putative auditory area that is located primarily ventral and anterior to RA (Kelley and Nottebohm, 1979; Vates et al., 1996; Mello et al., 1998; Yuan and Bottjer, 2019). We discuss the high degree of anatomic complexity in the arcopallial regions surrounding RA and Ald in a note to the paper by Mandelblat-Cerf et al. (2014). While such anatomic complexities remain to be clarified, we provide schematics of our recording locations in Figure $1 B$ to illustrate the region of arcopallium we refer to as Ald.

Throughout the text, we refer to Ald as analogous in function and connectivity to motor cortex, using the term "cortex" in a generic sense as described by Reiner et al. (2004; p. 395) as including the part of telencephalon that is "pallial in nature and therefore homologous as a field to the brain region of mammals that includes the neocortex, claustrum, and pallial amygdala."

\section{Electrophysiology}

At $35-40 \mathrm{dph}$, birds were anesthetized with $1.5 \%$ isoflurane (inhalation) and placed in a stereotaxic instrument. An electrode assembly consisting of four tetrodes affixed to a movable microdrive was fixed to the skull using C\&B Metabond (Parkell), such that the tetrodes were implanted $500 \mu \mathrm{m}$ dorsal to Ald. Each tetrode consisted of four twisted polyimide-coated Nichrome wires (0.012-mm diameter Redi Ohm 800, Kanthal) routed through fused silica capillary tubing and electroplated with non-cyanide gold plating solution (SIFCO 5355). One day after surgery, the tetrode assembly was connected to a recording headstage (HS-16, Neuralynx) with a flexible cable connected to a commutator (PSR, Neuralynx); 15 channels of neural data were amplified, band passed between 300 and $5000 \mathrm{~Hz}$ (Lynx-8, Neuralynx), and digitized at $32 \mathrm{kHz}$ using Spike2 software (Power 1401 data acquisition interface, CED). Audio and video were recorded coincident with neural activity: vocalizations were recorded with a lavalier microphone (Sanken COS-11D) mounted in the cage, and a USB-video camera (30 FPS, ELP) was placed at the

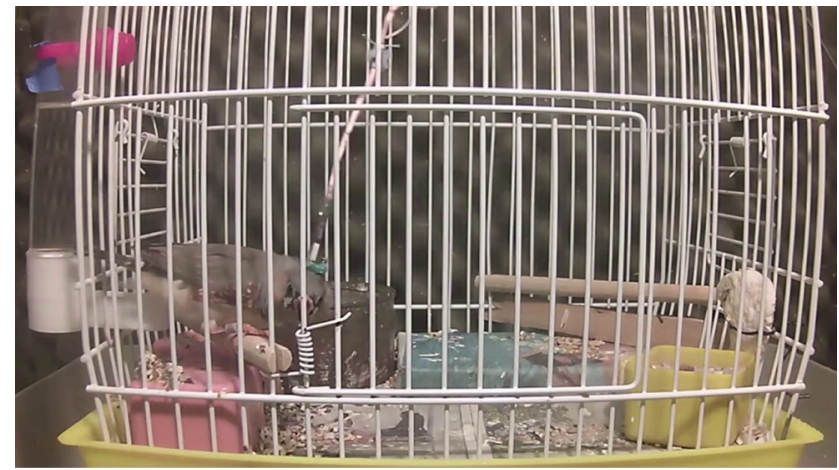

Movie 1. Example video of a juvenile zebra finch demonstrating the seven scored movements: pecks, beak interactions, preening, hopping, stretching, scratching, and fluff-ups. See Movie 2 for examples of pecking movements during eating versus noneating periods. [View online]

front of the cage to record video. Consecutive 30-min recordings were made from 7 A.M. to 6 P.M. each day. Tetrodes were manually advanced with the microdrive when the cells being recorded were lost or had already been recorded for at least $2 \mathrm{~d}$, as indicated by stability and consistency of the extracellular signal. At the end of each experiment, birds were perfused $(0.7 \%$ saline followed by $10 \%$ formalin), and brains were removed and postfixed for $72 \mathrm{~h}$ before being cryo-protected (30\% sucrose solution) and frozen-sectioned (50 $\mu \mathrm{m}$ thick). Sections were Nissl stained with thionin to visualize tetrode tracks and verify recording locations. Sites within $50 \mu \mathrm{m}$ from the border of Ald were considered to be within Ald if neural activity matched characteristic Ald firing (intermittent periods of high firing or a high rate of tonic activity during active behavior; Fig. 1C).

Movement artifact in neural recordings was correlated across recording channels and was eliminated or reduced using offline common average referencing: for each recording channel, the signal across $\sim 8-14$ remaining recording channels was averaged and subtracted from that channel to remove movement artifact (Ludwig et al., 2009). Channels were visually inspected after referencing to ensure that spiking activity was not distorted. After common average reference subtraction, single units were sorted from multiunit data by first automatically clustering units with KlustaKwik (KD Harris, University College London). KlustaKwik clusters were manually inspected across 18 different waveform features and further refined using MClust (A. D. Redish, University of Minnesota). Clusters were considered for analysis only if the signal-tonoise ratio was $>2$ and $<1 \%$ of spikes had an interspike interval (ISI) $<2 \mathrm{~ms}$.

\section{Behavioral scoring}

Sixteen 30 -min sessions were recorded across seven birds (median of three sessions per bird). Videos from recording sessions were scored for movements and state periods using ELAN (The Language Archive, Max Planck Institute for Psycholinguistics; Movies 1, 2, 3). We scored each single occurrence of pecks, hops, preening 


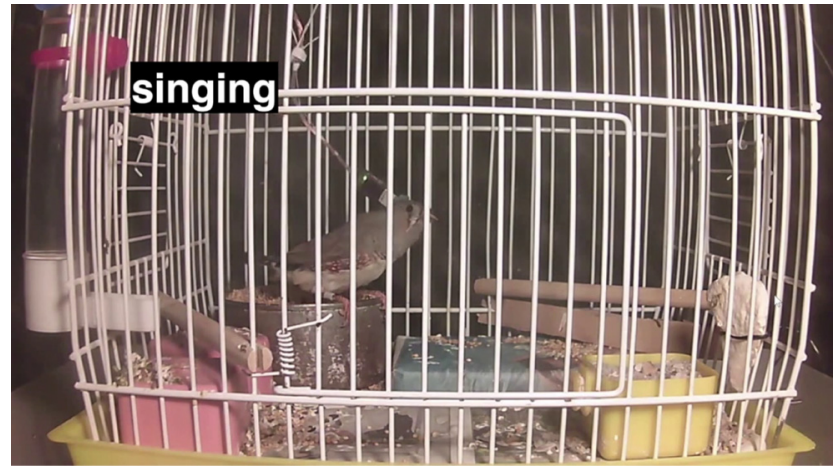

Movie 2. Example video of a juvenile zebra finch during periods of active behavior, singing, and eating. [View online]

behavior, beak interactions (beak wipes and periods when the bird's beak was in contact with perches, food cup edges, etc. for longer than the duration of a peck), fluff-ups, scratches, and stretches. Head and postural movements occurred so frequently that it was impractical to score all of them for all cells. These movements did not occur concurrently with any of the seven scored movements, but did occur during singing. To test whether head movements contributed to singing activity, we scored head movements that occurred during singing and during $30 \mathrm{~s}$ of non-singing before and after each singing episode
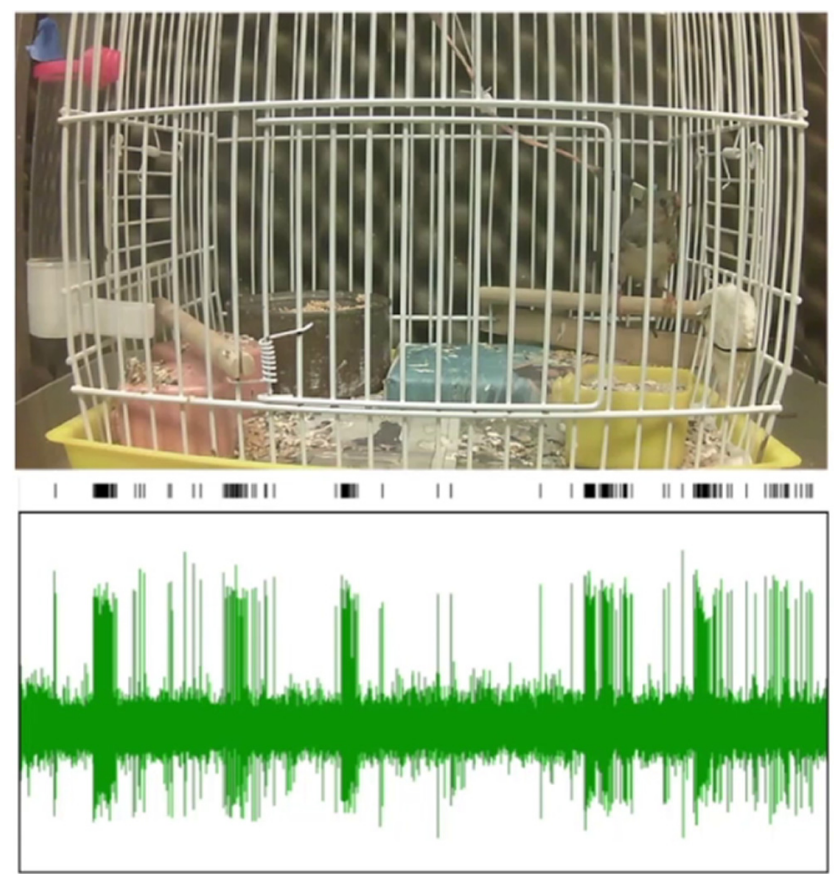

Movie 3. Example video of neural activity recorded on a single channel while the juvenile zebra finch hopped around the cage, demonstrating firing rate increases whenever the bird hopped towards the left side of the cage. This example suggests the possibility that hopping-related activity was context- or location-dependent, but we did not have enough examples to test this idea. Vertical lines above the raw activity trace indicate spikes from a single neuron sorted from the extracellular activity. [View online] in a subset of 36 singing-responsive neurons. In addition, all head and postural movements were scored for 12 neurons across two recording sessions in two birds.

In addition to scoring discrete movements, we developed a novel way of measuring behavior throughout recording sessions: each session was segmented into contiguous time periods that were classified into one of five behavioral "state" periods based on the bird's behavior: eating, singing, active-movement, quiet-attentive, or quiescent; these state periods tiled the entire duration of the recording session (Fig. 6A). Eating states were defined as periods during which the bird was pecking at seed or grit, hulling or ingesting seeds, or pausing in between these behaviors for at most $1 \mathrm{~s}$. Although pecks occurred most often during eating, eating states could also include other scored movements such as hops or preening, or unscored movements such as head movements as long as they occurred within the brief $(\leq 1 \mathrm{~s})$ pauses that occurred while birds were actively eating. Singing states were defined by song behavior; they began whenever the bird produced song and lasted as long as song syllables continued to occur within $1 \mathrm{~s}$ of each other (intersyllable interval $\leq 1 \mathrm{~s}$ ). Birds often made head movements during singing and occasionally made scored movements such as hopping or pecking in between bouts of singing. Active-movement states were defined as non-eating and non-singing periods during which the bird made active movements with pauses of at most $1 \mathrm{~s}$ in between movements; these periods could include any of the seven movements that were scored as well as head and postural body movements that were not scored. Quiet-attentive states were defined by times when the bird was not eating, singing, or moving around the cage for $>1 \mathrm{~s}$; they continued as long as the bird made at most small head movements and otherwise remained unmoving but alert. Quiescent periods were defined as periods during which the bird was completely still and not obviously paying attention to any stimulus, with eyes partially or fully closed. Quiescent state times were segmented into 1-s intervals that were used as baseline periods for analyses of scored movements (see "Data analysis" section below).

\section{Data analysis}

To test for significant responses during scored movements, firing rates across occurrences of each movement type were compared against quiescence. Quiescent baseline periods were generated by dividing quiescent state periods (as described in "Behavioral scoring" section above) into 1-s segments. The firing rate during two 1 -s quiescent segments that occurred closest in time to each movement occurrence was used as a corresponding baseline. Fourteen neurons were recorded during sessions that lacked quiescent state periods. For these 14 neurons, 1-s baseline periods were taken from times within quiet-attentive state periods when the bird was verified to be unmoving (although clearly alert, unlike in quiescent state). To compare movement responses across neurons, standardized response strength (RS) was calculated for each movement type as: 


\section{standardized response strength}

$$
=\frac{\overline{F R}_{m}-\overline{F R}_{b}}{\sqrt{\operatorname{Var}\left(F R_{m}\right)+\operatorname{Var}\left(F R_{b}\right)-2 \times \operatorname{Covar}\left(F R_{m}, F R_{b}\right)}},
$$

where $\mathrm{FR}_{\mathrm{m}}$ is the firing rate during movement occurrences and $F R_{b}$ is the firing rate during corresponding baseline periods. A positive value indicates an increase in firing rate during the movement compared with quiescence whereas a negative value indicates a decrease in firing rate during movement. This measure is referred to as RS throughout the text. Mean RS values across neurons are reported as the mean \pm SEM.

To test for changes in activity around movement onsets, for each neuron we generated a 25-ms bin histogram of the spiking response across all occurrences of the movement; histogram windows were $1 \mathrm{~s}$ long and centered on movement onsets. Spike times during each movement repetition were shuffled to obtain a resulting histogram of shuffled spike data; this was repeated 1000 times, resulting in 1000 histograms of shuffled data. Each bin of the actual spike data histogram was considered significantly excited if the count in that bin was $>95 \%$ of maximum values from the shuffled dataset; likewise, the bin was considered significantly suppressed if the count was lower than $95 \%$ of minimum values from the shuffled dataset. Onset responses were defined as responses that contained at least two consecutive bins $(50 \mathrm{~ms})$ of significant maxima or minima within $100 \mathrm{~ms}$ of movement onset.

We tested for significant offset-aligned responses using the same method and parameters as onset-aligned responses, except that the 1-s windows used for histograms of actual and shuffled data were centered around movement offsets; offset responses were defined as responses that contained at least two consecutive bins (50 ms) of significant maxima or minima within $100 \mathrm{~ms}$ of movement offset. Because of the short duration of pecks and hops (mean hop duration $=0.28 \mathrm{~s}$; mean peck duration $=0.25 \mathrm{~s}$ ), it was possible for the same maxima or minima to be captured in both onset-aligned and offsetaligned responses. To ensure that offset-aligned activity could be accurately distinguished, for these movements, we only counted excited or suppressed offset responses that did not demonstrate significant changes in onsetaligned activity. Preening movements were relatively long in duration (mean duration $=1.5 \mathrm{~s}$ ), so all preening-aligned offset responses were counted.

We tested for significant modulation of firing rate during states by dividing all state periods in each recording session into 1-s segments and calculating the average firing rate during each segment for each neuron. We compared the distributions of average firing rates across segments for each state against quiescence to determine whether activity was significantly increased or decreased during non-quiescent states for each neuron. Fourteen neurons were recorded during sessions that lacked quiescent state periods and were excluded from these analyses.

As an additional means of characterizing firing rate modulation during states, we defined "events," brief periods of excitation and suppression, from histograms of spiking activity. We segmented the instantaneous firing rate (IFR) across each recording session into 10-ms bins and smoothed the IFR with a moving average filter (span $=3$ bins). Excited events were defined as periods during which the smoothed IFR across five or more 10-ms bins (50 ms or more) exceeded the average firing rate across quiescent state periods by $\geq 3$ SDs. Suppressed events were defined as periods during which the smoothed IFR across five or more 50 -ms bins fell below the average firing rate across quiescent state periods by $\leq 1.5$ SDs. To compare across neurons, the number of events in each state was normalized by dividing the number of excited or suppressed events in each state by the total duration of that state for each neuron.

\section{Statistics}

Movement responses were tested for significance against quiescent baselines (see "Data analysis" section above) using Wilcoxon signed-rank tests; Benjamini-Hochberg post hoc tests were used to apply corrections for multiple comparisons (Benjamini and Hochberg, 1995). Neurons that demonstrated a significant difference between movement and baseline for at least one scored movement were considered movement-responsive. To test whether movement responses were context-selective, RSs during movements from one context versus another context were compared using Mann-Whitney tests for each neuron (for example, comparing pecks during eating vs non-eating periods, and comparing head movements during singing vs non-singing periods). Mann-Whitney tests were also used to compare RSs during singing periods with versus without head movements in individual neurons. We were not able to use signed-rank tests in these cases because of different numbers of observations in the comparisons. $\chi^{2}$ tests were run to compare proportions between more than two groups (for example, proportions of neurons that were responsive during each movement type). In case of significance, Fisher's exact tests were used as a follow-up to make pairwise comparisons of proportions between groups, and BenjaminiHochberg post hoc tests were used to apply a correction for multiple comparisons. Binomial tests were used to judge whether the relative proportions of excited versus suppressed responses among movement-responsive neurons were different from chance. Comparisons of firing rate distributions between each state and quiescence (see "Data analysis" section above) were made using KolmogorovSmirnov tests, with a Benjamini-Hochberg post hoc test applied for multiple comparisons. Measures between different state periods (ISIs, normalized number of events, normalized firing rates among unresponsive neurons) were compared using sets of pairwise linear contrasts based on trimmed means (20\% trimming); this linear contrast method has been shown to be robust to common assumption violations such as non-normality and heteroscedasticity (Wilcox and Serang, 2017). For all tests, $p<0.05$ was considered significant.

\section{Results}

We made extracellular recordings from 119 neurons in Ald of freely behaving juvenile zebra finches (43-58 dph) 
housed singly in a recording cage as they actively engaged in sensorimotor vocal practice. Figure $1 B$ illustrates locations of recording sites in Ald. A typical 30-min recording period included various overt behaviors and periods of quiescence when the bird was not moving. To investigate how neural activity in Ald corresponds to different behaviors, we scored seven different movements during each recording that could be identified reliably: pecks, hops, preening episodes, beak interactions with objects in the recording cage (e.g., beak wipes or nonpeck interactions with cage bars), fluff-ups, stretches, and scratching episodes; we also marked periods of singing (Movies 1, 2, 3). We developed a novel approach in which we examined spiking patterns of single neurons throughout each recording period to investigate whether Ald neurons are selective for different movement types and/or singing behavior in juvenile birds.

\section{Responsivity of Ald neurons during movements}

Patterns of spiking were highly variable across individual neurons, ranging from phasic to tonic activity. In addition, each neuron's activity was highly modulated throughout a typical recording session, showing either excitation and/or suppression during different movements. Figure $1 \mathrm{C}$ illustrates two different neurons recorded in one bird while it was quiescent (no overt movements, left columns) and while it hopped around the cage (right columns). The neuron in the top panel fired intermittently in small bursts of at most three spikes during quiescence, while the neuron in the bottom panel exhibited dense bursting activity. As the bird hopped around the cage, the neuron in the top panel shifted to longer periods of high firing separated by relative inactivity while the neuron in the bottom panel shifted to a high tonic rate of firing. To investigate whether such modulations were related to specific movements, for each neuron we compared firing rates during different movement types against baseline firing rates during quiescent periods that were closest in time to each movement occurrence (see Materials and Methods). To compare movement-related activity across neurons, we calculated the response strength of each neuron during each movement type, defined as the standardized difference in average firing rate during each movement type versus baseline periods (see Materials and Methods).

The majority of Ald neurons exhibited a significant change in firing rate during at least one movement type compared with quiescent baseline periods and were thus classified as "movement responsive" (101 out of 119 neurons, 85\%). Among 101 movement-responsive neurons, $33(33 \%)$ responded during only one scored movement, whereas $68(67 \%)$ responded during two or more movements (Fig. 2A). Few cells responded during five or six movements, and no cells responded during all seven movements. Figure $2 B$ depicts the range of responsivity in these 101 neurons. For example, 10 neurons were either excited or suppressed during pecks but were otherwise not responsive during any of the other six scored movements; likewise, two other groups of 10 neurons each selectively modulated their firing rate only during preening or hops. Neurons modulated during multiple movements showed heterogeneous responsivity: single neurons could demonstrate excitation during some movement types and suppression during others, and responsivity profiles across neurons included different subsets of movement types.

Higher proportions of neurons showed altered firing rates during pecks, preening, and/or hops compared with other movements: 68 out of 107 neurons (64\%) were significantly modulated during pecks, 50 out of 93 (54\%) during preening, and 60 out of 119 (50\%) during hops (Fig. $2 C)$. These proportions did not differ (Fisher's exact test, Benjamini-Hochberg corrected, peck vs preen $p=0.23$, peck vs hop $p=0.09$, preen vs hop $p=0.71$ ) and were each greater than the proportions of neurons that responded during fluff-ups $(21 / 79,27 \%)$, scratches $(14 / 55$, $25 \%)$, and stretches (7/59, 12\%; Fisher's exact test, Benjamini-Hochberg corrected, $p<0.05$ for comparisons between pecks, hops, and preening against each of the other three movements; $p>0.05$ for comparisons among these latter three movements; Fig. 2C). In addition to the seven scored movements, birds constantly made quick, saccade-like movements throughout recording periods, resulting in over a thousand head or postural movements in a typical 30-min session. As an initial test of whether firing rate was modulated during these latter movements, we scored all head and postural movements for a subset of 12 neurons and found that six neurons were excited during these movements $(50 \%)$, while four were suppressed (33\%), demonstrating that Ald activity can be modulated during head and postural movements as well.

As indicated above, we observed both excited and suppressed responses within single neurons: 21 out of 68 neurons $(31 \%)$ that responded during multiple movements exhibited excitation during some movements and suppression during others (Fig. 2B). The overall proportions of excited versus suppressed responses did not differ (56\%, 138/247 excited; 44\%, 109/247 suppressed; binomial test, $p=0.07$ ), indicating a fairly even representation of excitation and suppression across scored movements. In addition, the proportions of excited versus suppressed responses during each movement type did not differ (binomial test, $p>0.05$ in all cases; Fig. 2C).

\section{Temporal response patterns of Ald neurons at movement onsets and offsets}

Some Ald neurons demonstrated consistent temporal changes in firing rate at movement onsets and/or offsets that could be masked by measures of average firing rate. For example, Figure $3 A$ shows rasters and histograms for a single neuron during preening (Fig. $3 A$, left) and peck (Fig. $3 A$, right) responses. In both cases, mean firing rate during the movement was significantly excited relative to quiescence (preening $\mathrm{RS}=0.65$, peck $\mathrm{RS}=1.53, p<0.05$ in both cases). However, the responses clearly contain periods of suppression that begin before movement onset.

To capture these firing rate modulations, we tested for significant excitation or suppression at movement onsets and offsets. For each response, we compared histograms of spiking activity centered on movement onsets or 
A

number of movement types responded to

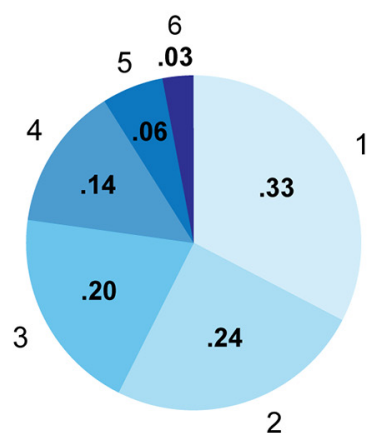

\begin{tabular}{r} 
\# movement \\
\hline \\
1 \\
2 \\
3 \\
4 \\
5 \\
6
\end{tabular}
B

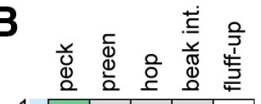

1
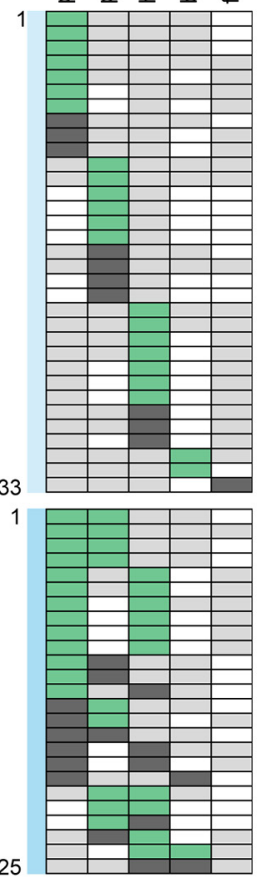
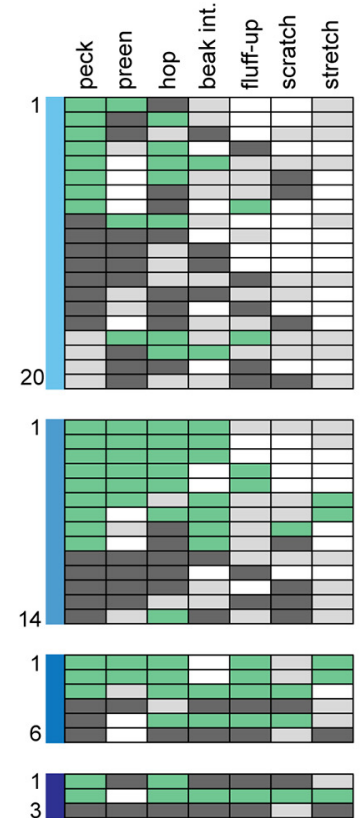

excited

suppressed

no response

C

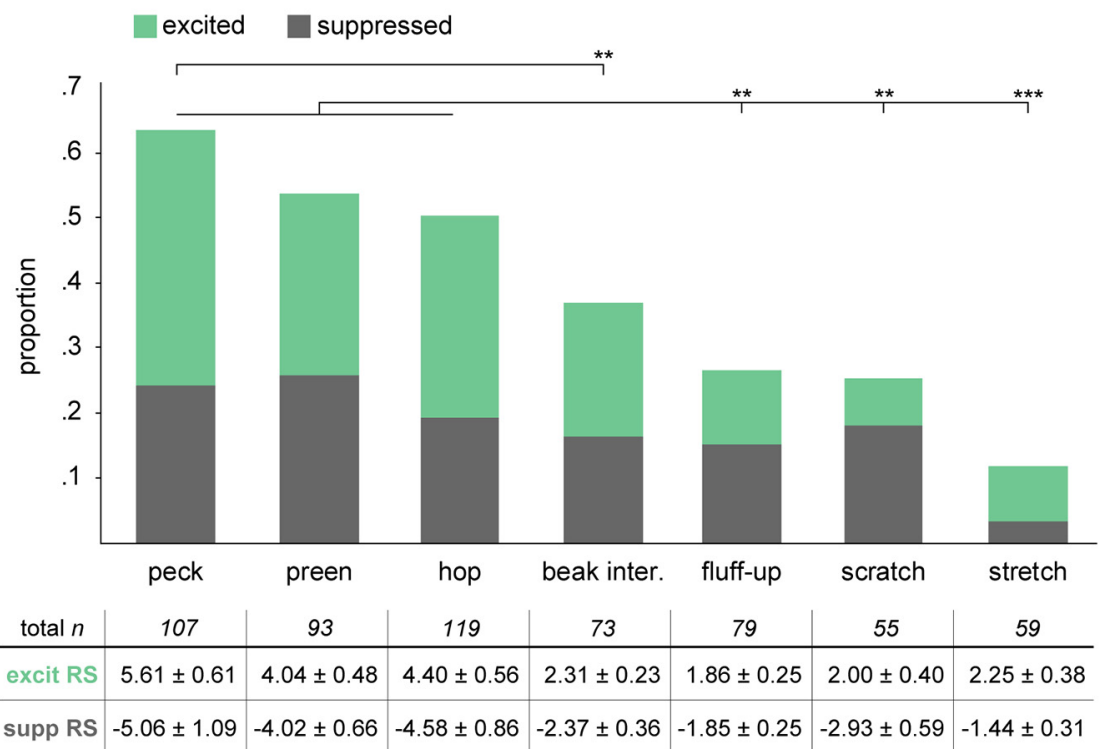

Figure 2. Ald neurons respond during different scored movements with excitation and/or suppression. A, Proportions of single Ald neurons that responded during different numbers of movements. 33/101 movement-responsive neurons responded during one movement, 25/101 during two, 20/101 during three, 14/101 during four, 6/101 during five, and 3/101 during six movements. $\boldsymbol{B}$, Each row of each chart indicates movements during which each neuron was excited (green), suppressed (dark gray), or not responsive (light gray). Unfilled boxes indicate that no data during that movement was recorded for that neuron. Charts are grouped according to colors in $\boldsymbol{A}$, based on the number of movements during which neurons responded. $\boldsymbol{C}$. Proportions of Ald neurons that were significantly excited (green) or suppressed (dark gray) during each movement type. Table below indicates the number of neurons recorded during each movement type and the corresponding excited and suppressed RSs (mean standardized RS \pm SEM); $* * p<0.005, * * * p<0.0001$.

offsets to histograms of shuffled spike trains (25-ms bins) to identify bins with significant firing rate changes (see Materials and Methods). Onset or offset responses were defined as responses with two or more contiguous bins (50 ms) of significant excitation or suppression occurring within $100 \mathrm{~ms}$ of movement onset or offset. Figure 3 plots examples of onset-aligned (Fig. $3 A-C$, left) and offsetaligned (Fig. $3 C$, right) responses; green and gray horizontal bars above each histogram mark excited and suppressed bins, respectively. 
A
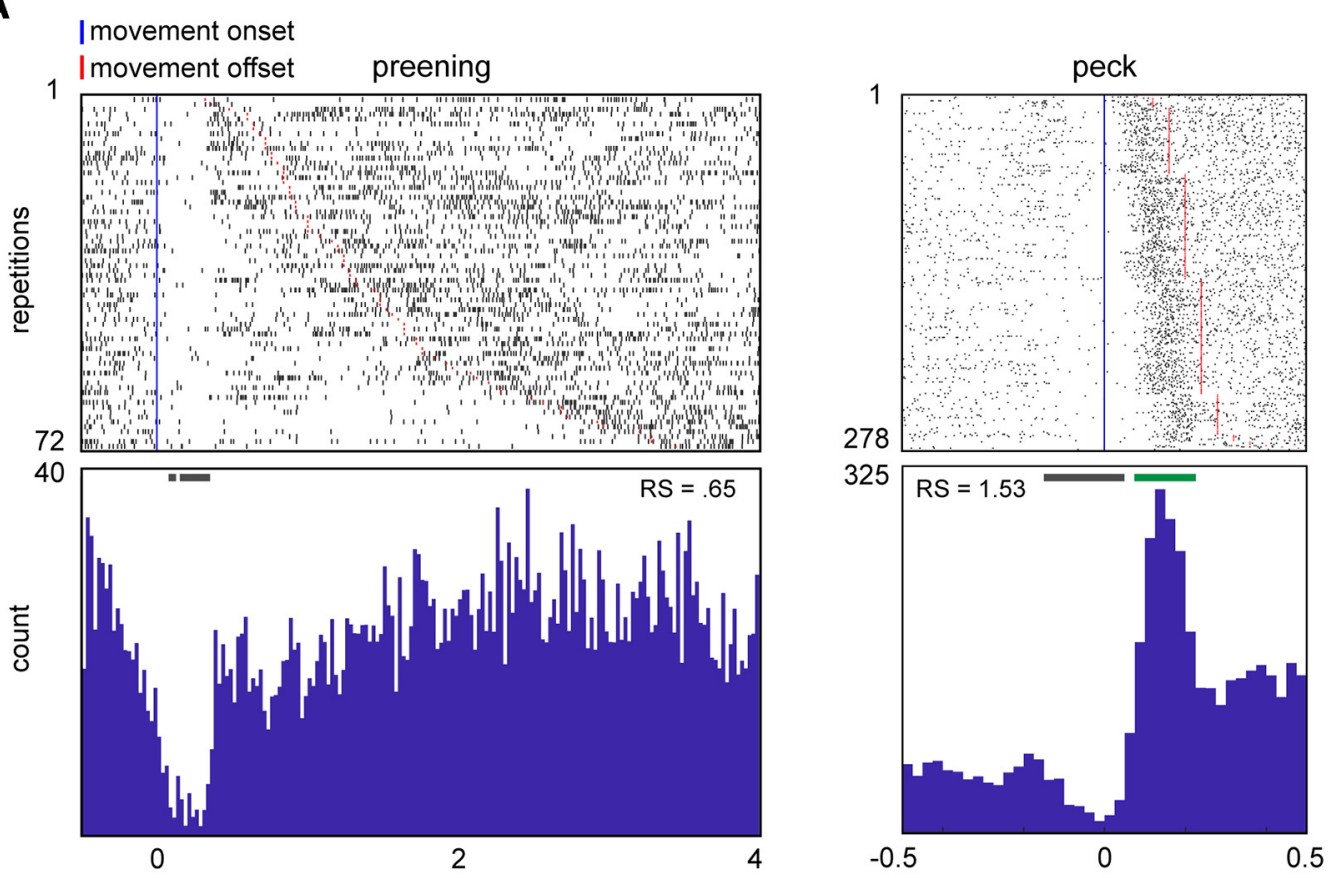

time from preening onset (sec)

time from peck onset (sec)

B

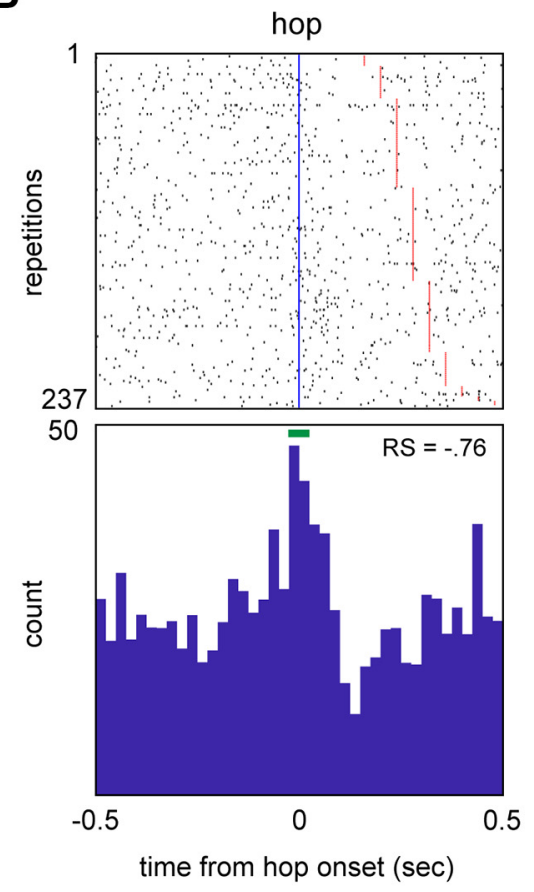

C

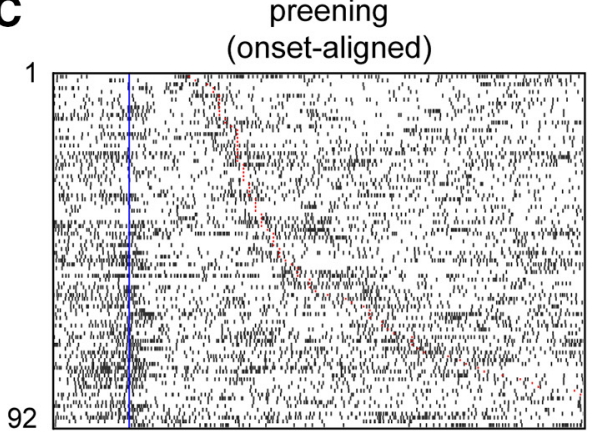

85

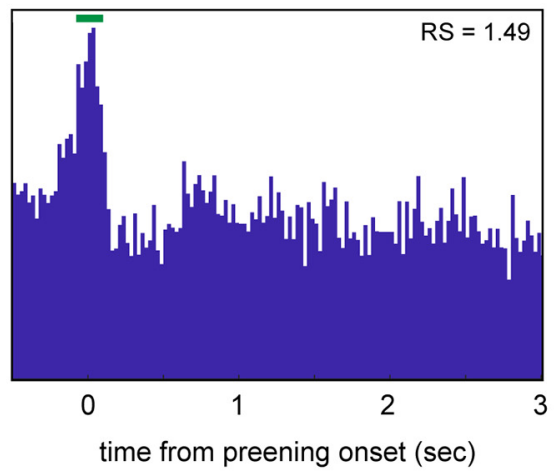

preening

(offset-aligned)
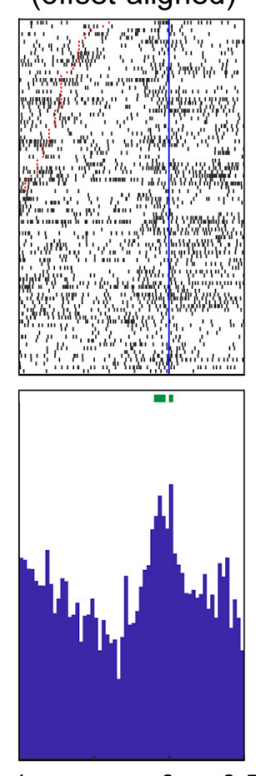

$\begin{array}{ll}0 & 0.5\end{array}$

time from

preening offset (sec)

Figure 3. Ald neurons show a variety of temporal response patterns during different scored movements. $\boldsymbol{A}$, Rasters and histograms illustrating the response of a single Ald neuron during preening (left) and pecks (right). $\boldsymbol{B}$, Raster and histogram illustrating the response of a single Ald neuron during hops. $\boldsymbol{C}$, Rasters and histograms illustrating the onset-aligned (left) and offset-aligned (right) preening response of an example Ald neuron. Rows are sorted by movement duration. Blue vertical lines mark movement onsets; red lines mark movement offsets. Green and gray horizontal bars above histograms denote periods of excitation or suppression, respectively (see Materials and Methods). RS, average standardized response strength over the entire duration of each movement type. 
Table 1: Proportions of onset and offset responses across all neurons for different movement types

\begin{tabular}{|c|c|c|c|c|c|c|}
\hline \multirow{2}{*}{$\begin{array}{l}\text { Response based } \\
\text { on average } \\
\text { firing rate }\end{array}$} & \multicolumn{2}{|c|}{$\begin{array}{c}\text { Peck-onset responses } \\
\text { ( } \pm 100 \text { ms from peck onset) }\end{array}$} & \multicolumn{2}{|c|}{$\begin{array}{l}\text { Hop-onset responses } \\
\text { ( } \pm 100 \text { ms from hop onset) }\end{array}$} & \multicolumn{2}{|c|}{$\begin{array}{l}\text { Preening-onset responses } \\
\text { ( } \pm 100 \text { ms from preening } \\
\text { onset) }\end{array}$} \\
\hline & $\begin{array}{c}\text { Excited } \\
(7) \\
\end{array}$ & $\begin{array}{c}\text { Suppressed } \\
(4)\end{array}$ & $\begin{array}{c}\text { Excited } \\
(4) \\
\end{array}$ & $\begin{array}{c}\text { Suppressed } \\
(1)\end{array}$ & $\begin{array}{c}\text { Excited } \\
(3)\end{array}$ & $\begin{array}{c}\text { Suppressed } \\
(1)\end{array}$ \\
\hline Excited & $0.05(5 / 107)$ & $0.04(4 / 107)$ & $0.01(1 / 119)$ & 0 & $0.03(3 / 93)$ & $0.01(1 / 93)$ \\
\hline Not significant & $0.02(2 / 107)$ & 0 & $0.02(2 / 119)$ & 0 & 0 & 0 \\
\hline & \multicolumn{2}{|c|}{$\begin{array}{l}\text { Peck-offset responses } \\
\text { ( } \pm 100 \text { ms from peck offset) }\end{array}$} & \multicolumn{2}{|c|}{$\begin{array}{l}\text { Preening-offset responses } \\
\text { ( } \pm 100 \text { ms from preening } \\
\text { offset) }\end{array}$} & & \\
\hline $\begin{array}{l}\text { Response based } \\
\text { on average } \\
\text { firing rate }\end{array}$ & $\begin{array}{l}\text { Excited } \\
\text { (4) }\end{array}$ & $\begin{array}{l}\text { Suppressed } \\
\text { (2) }\end{array}$ & $\begin{array}{l}\text { Excited } \\
\text { (3) }\end{array}$ & $\begin{array}{l}\text { Suppressed } \\
\text { (0) }\end{array}$ & & \\
\hline
\end{tabular}

Onset responses (top) and offset responses (bottom) are shown separately (total $n=29$ responses), categorized based on whether average firing rate during the movement showed significant excitation (excited), suppression (suppressed), or no response (not significant).

We observed significant onset responses during pecking, hopping, and preening but not other movement types. The top of Table 1 lists onset responses for these three movements, classified by whether neurons showed a significant response based on average firing rate. Eleven out of 107 neurons (10.3\%) exhibited significant excitation (7/11) or suppression (4/11) at peck onsets. Five out of 119 neurons demonstrated an onset response during hopping (4.2\%; 4/5 excitation, $1 / 5$ suppression), as did four out of 93 neurons during preening (4.3\%; $3 / 4$ excitation, $1 / 4$ suppression). We also observed significant offset responses during pecking and preening: six out of 107 neurons exhibited pecking offset responses (5.6\%; 4/6 excitation, 2/6 suppression), and three out of 93 neurons showed preening offset responses (3.2\%; all excitation; Table 1, bottom).

A total of 13 of these 29 onset and offset responses did not match the average firing rate response (Table 1). For example, four responses (two pecking, two hopping) included consistent excitation at movement onset even though average firing rate during movement did not differ from quiescent baseline (Table 1, top, "not significant" row). Onset and offset responses could also differ in sign (excitation or suppression) from the average firing rate response: for example, while average firing rate during the hop response plotted in Figure $3 B$ was suppressed relative to baseline $(R S=-0.76)$, the raster and histogram reveal an excitatory peak starting just before hop onset, indicating a complex temporal response with brief excitation followed by suppression. These results suggest that single Ald neurons can be modulated by multiple factors during movements, resulting in excitation at movement onsets or offsets and suppression during the movement itself, or vice versa.

\section{Context dependency of pecking behavior}

Of the seven movements we scored, pecking behavior in particular tended to occur in different contexts: birds always pecked while eating, but also frequently pecked at other objects such as cage bars or perches. To investigate whether different contexts influenced responsivity, we compared RSs for pecks that occurred during eating versus non-eating.

RS differed for eating- versus non-eating pecks in 47 out of 97 neurons (48\%; Mann-Whitney test, $p<0.05$ ). Figure $4 A$ plots these context-sensitive cells according to whether they exhibited greater absolute RS during eating (29/47, $62 \%$; left) or non-eating (18/47, $38 \%$; right). Figure $4 A$, left panel, shows 29 neurons that exhibited greater absolute RS during eating-related pecks. Most of these neurons showed higher firing rates during eating-related pecks compared with non-eating pecks $(21 / 29,72 \%$; gray lines). The peckaligned activity for one of these neurons (Fig. 4B) illustrates strong excitation during pecks that occurred when the bird was eating (left) versus a weak response during non-eating (right). The remaining neurons showed lower firing rates during eating-related pecks compared with non-eating pecks (8/29, 28\%; black lines). In contrast, Figure $4 A$, right panel, plots 18 neurons that showed greater absolute RS during non-eating pecks. Eleven of these neurons showed higher firing rates during non-eating pecks compared with eating pecks $(61 \%$, gray lines); the remaining cells showed lower firing rates during non-eating pecks $(7 / 18,39 \%$, black lines).

These results indicate that pecking activity in many Ald neurons was dependent on the context in which the movement occurred and suggest that neurons can signal behavioral contexts with either relative excitation or suppression. While peck duration did not differ between eating versus non-eating (mean peck duration $=0.24 \pm 0.002$ vs $0.22 \pm$ $0.003 \mathrm{~s}$, respectively), one possibility is that this context-dependent activity reflects differences in eating versus noneating pecking movements. Alternatively, these differential responses may reflect that these neurons do not encode the physical movements of pecking behavior per se; for example, this subpopulation may be involved in processing orofacial or external sensory information that is present specifically in one context versus another. 
A
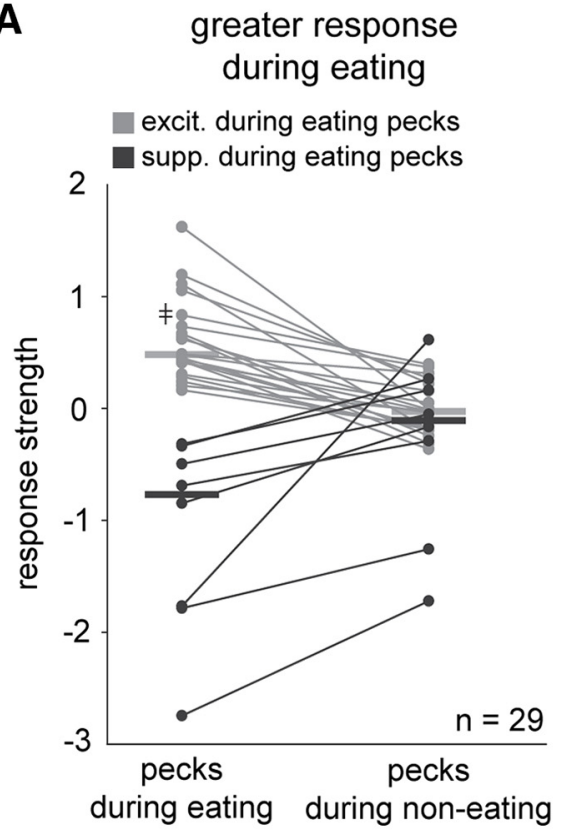

B $^{\neq}$
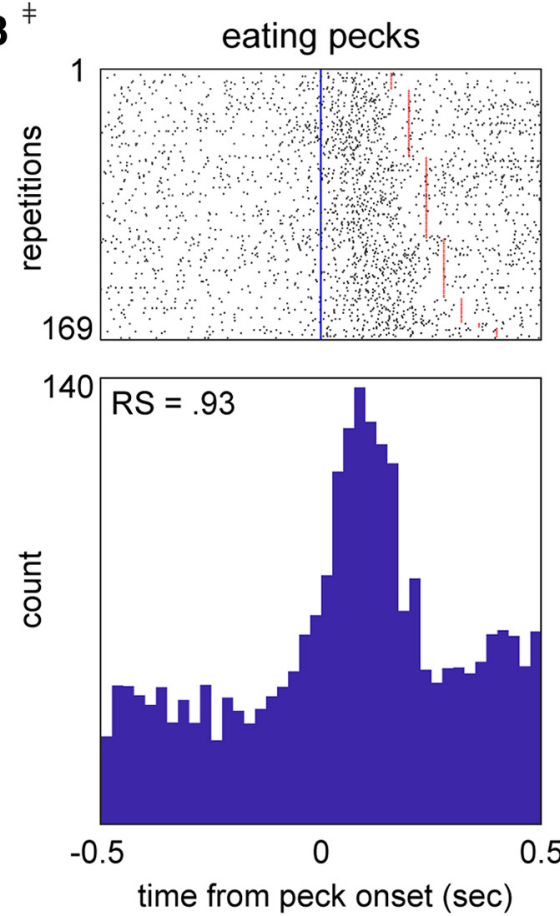

greater response during non-eating
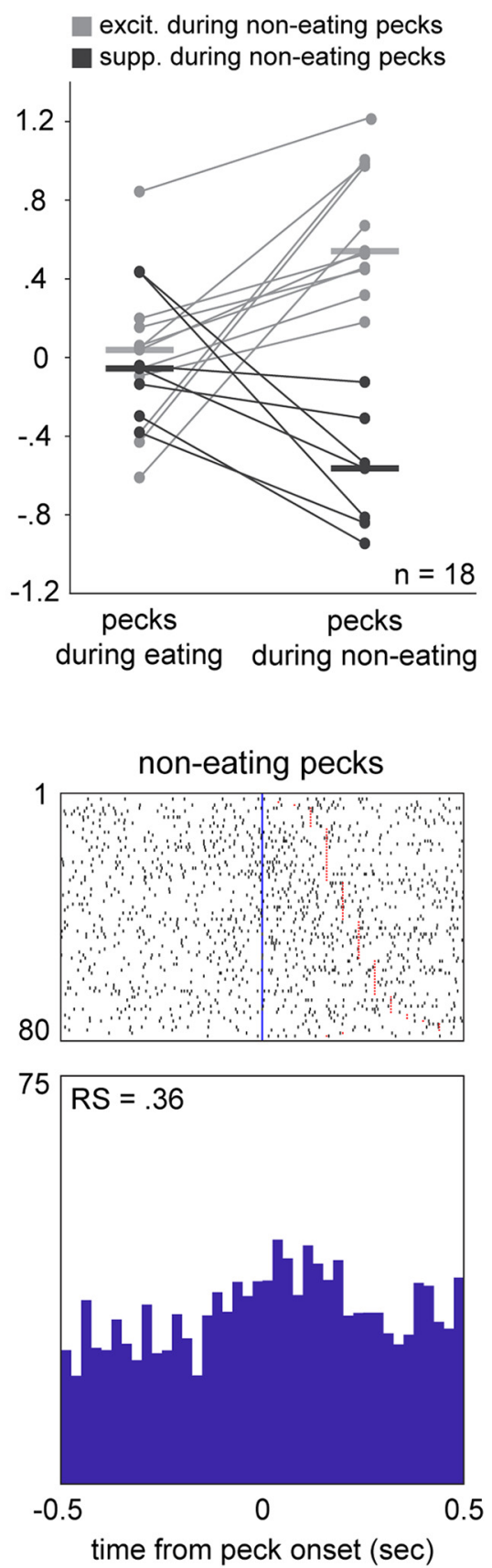

Figure 4. Ald neurons exhibit context-sensitive peck responses. $\boldsymbol{A}$, Mean standardized RSs of neurons during pecks that occurred during eating versus non-eating periods, grouped by neurons that showed greater absolute RS during eating (left) and non-eating (right). Left, Gray and black lines represent neurons that showed positive or negative RS, respectively, during eating-pecks. Right, Gray and black lines represent neurons that showed positive or negative RS, respectively, during non-eating pecks. Horizontal bars represent medians. RSs during eating-related pecks were significantly different from non-eating pecks for all plotted neurons (Mann-Whitney tests). B, Rasters and histograms of an example neuron's response during pecks that occurred during eating (left) versus non-eating (right). Peck RS of this neuron is indicated by the cross-marked plot point in $\boldsymbol{A}$, left. Rows are sorted by peck duration. Blue vertical lines mark peck onsets; red lines mark peck offsets.

\section{Singing-responsive neurons in Ald}

One of Ald's primary sources of afferents is from LMAN-SHELL, which contains neurons that are active during singing behavior and have been implicated in guiding accurate imitation of the tutor song during vocal learning (Fig. 1A; Achiro and Bottjer, 2013; Achiro et al., 2017). Moreover, lesions of Ald in juvenile birds impair their ability to achieve an accurate imitation of the adult tutor song 


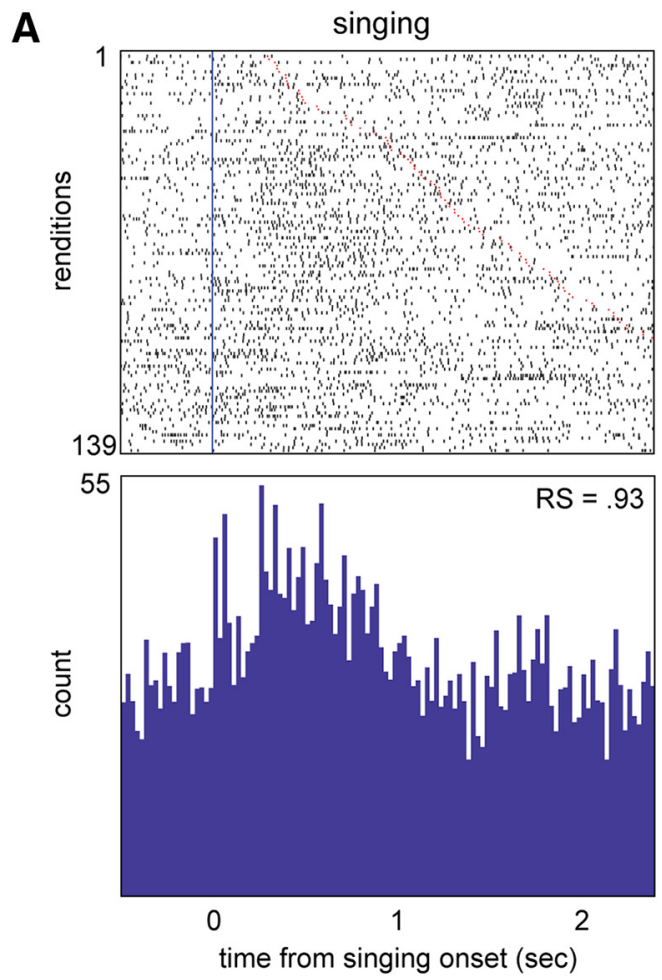

B singing-responsive neurons
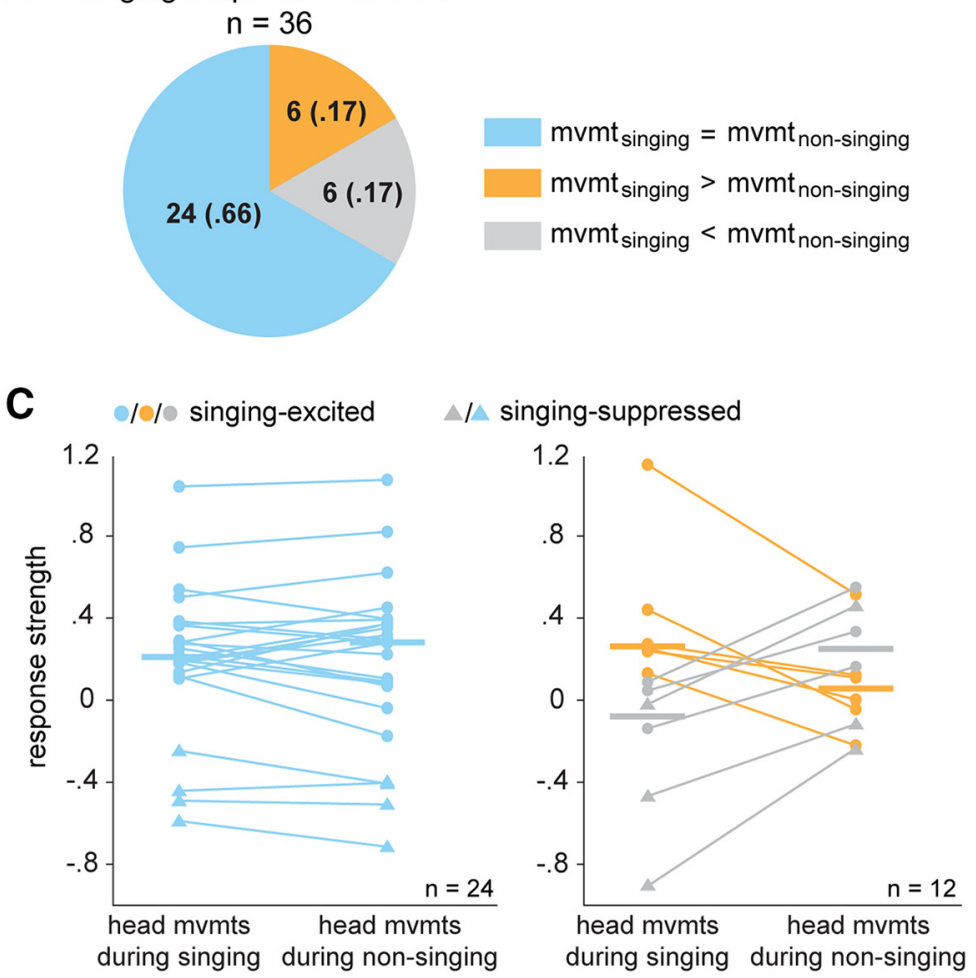

Figure 5. A substantial population of Ald neurons are responsive during singing. A, Raster and histogram illustrating activity of an example singing-excited Ald neuron during singing episodes. Rows are sorted by duration of each singing episode. Blue vertical line marks onset of each singing episode; red lines mark ends of singing episodes. $\boldsymbol{B}$, Proportions of 36 singing-responsive neurons for which RS during head movements within singing periods was greater than (orange), less than (gray), or not different from (blue) head movements within non-singing periods. $\boldsymbol{C}$, Mean standardized RSs during head movements that occurred within singing versus non-singing periods. Left, Neurons that showed comparable RS during head movements that occurred within singing versus non-singing periods. Right, Neurons that showed higher RS during head movements that occurred within singing (orange) or non- 
continued

singing (gray) periods. Lines connect data points from single neurons. Horizontal bars represent medians. Circles versus triangles represent neurons that showed an increase or decrease, respectively, in average firing rate across singing episodes relative to quiescence (see Tables 2-4).

without disrupting vocal motor output (Bottjer and Altenau, 2010; see Mandelblat-Cerf et al., 2014; Materials and Methods). Given this evidence of a role for Ald in vocal learning, we hypothesized that the activity of Ald neurons would be modulated as juvenile birds engaged in singing behavior.

Firing rates were significantly modulated during singing relative to quiescence in the majority of neurons $(66 / 94$, $70 \%$ ), including 44 excited responses and 22 suppressed responses (mean RS $=0.76 \pm 0.09$ and $-0.96 \pm 0.19$, respectively). Figure $5 \mathrm{~A}$ illustrates the singing-aligned response of a neuron that was excited during song renditions. Altered firing rates during vocal production in songbirds have typically been interpreted as "singing specific." However, birds often make head and postural body movements during singing, as well as beak-gape and gular-fluttering movements that are specific to song production. This complexity raises the question of which movements are an intrinsic part of singing behavior versus independent movements that are performed simultaneously during song production. Given the range of movement responsivity across Ald neurons (Fig. 2), activity modulation in singing-responsive neurons may reflect singing-specific actions as well as movements that are performed during both singing and non-singing periods. As an initial test of this question, we compared neural activity during head movements that occurred within singing periods versus adjacent non-singing periods in a subset of 36 singing-responsive neurons. We then compared activity during singing periods with versus without head movements to assess whether head movements contributed to the singing response in these neurons (see Materials and Methods).

RS during head movements that occurred within singing versus non-singing periods did not differ for most singing-responsive neurons (24/36, 67\%; Mann-Whitney test, $p>0.05$ for each neuron; Fig. 5B,C, left). In eight of these 24 neurons, RS during singing periods that contained head movements was significantly greater than during singing that lacked head movements, indicating that activity during head movements contributed to the singing response (7/8 excited, 1/8 suppressed; Table 2, left). For the remaining 16 neurons, RS during singing that lacked head movements either did not differ from (14/24) or was greater than $(2 / 24)$ RS during singing with head movements (Table 2, middle and right). In addition, most of these 24 neurons still showed significant changes in firing rates during singing that lacked head movements (21/ $24,88 \%)$. Thus, firing rate changes in most of these singing-responsive neurons was not attributable to activity during head movements.

Six neurons were singing excited and showed greater RS during head movements that occurred within singing compared with non-singing (Fig. 5B,C, right, orange). In three of these neurons, activity during singing periods with head movements was greater than during singing without head movements, indicating that singing-specific head movements contributed to excitation during song production (Table 3, left). For the other three neurons, responses during singing periods with versus without head movements were comparable, suggesting that discrete head movements made little contribution to activity modulation during singing (Table 3 , middle). Moreover, in all but one of these six neurons, activity during singing periods that lacked head movements was still significantly greater than quiescence.

Three neurons were singing suppressed and showed lower RS during singing-related head movements compared with non-singing (Fig. $5 \mathrm{C}$, right, gray triangles). For two of these neurons, decreased firing rates during head movements contributed to greater suppression during singing (Table 4, left, suppressed). However, all three neurons were suppressed even during singing periods that lacked head movements. In fact, for one of these neurons, this suppression was significantly greater than during singing that contained head movements (Table 4, right, suppressed). Interestingly, three neurons showed lower RS during singing-related head movements but

Table 2: Mean standardized RS during singing periods with versus without head movements ( $n=24$ neurons that showed comparable firing rates during head movements that occurred within singing and non-singing periods)

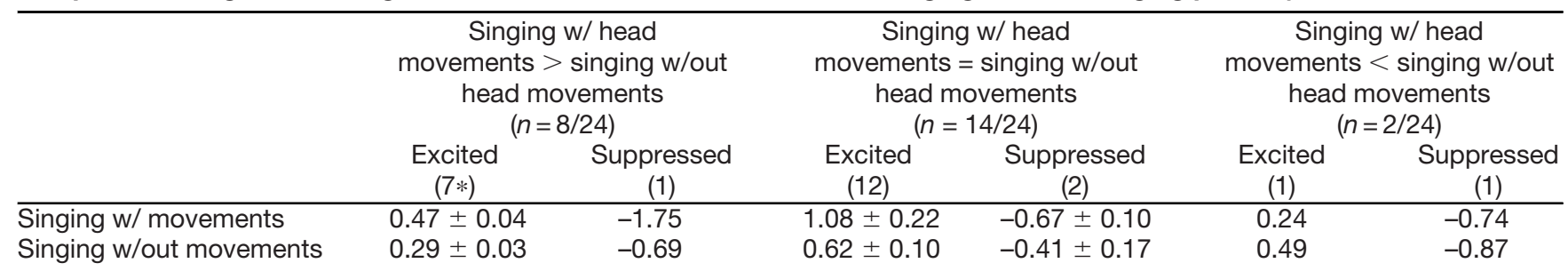

Neurons are categorized by whether RS during singing that included head movements was greater than (left), equal to (middle), or lower than (right) RS during singing that lacked head movements. Responses are significantly different from quiescence unless otherwise noted $\left(^{\star}\right)$. Blue lines in Fig. $5 \mathrm{C}$, left, depict head movement responses.

* 3/7 neurons were significantly excited during singing periods that contained head movements but not during singing periods that lacked head movements. 
Table 3: Mean standardized RS during singing periods with versus without head movements ( $n=6$ neurons that showed greater RS during head movements that occurred within singing compared with non-singing periods)

\begin{tabular}{|c|c|c|c|c|c|c|}
\hline & \multicolumn{2}{|c|}{$\begin{array}{c}\text { Singing } \mathrm{w} / \text { head } \\
\text { movements }>\text { singing w/out } \\
\text { head movements } \\
(n=3 / 6)\end{array}$} & \multicolumn{2}{|c|}{$\begin{array}{c}\text { Singing } \mathrm{w} / \text { head } \\
\text { movements }=\text { singing w/out } \\
\text { head movements } \\
(n=3 / 6)\end{array}$} & \multicolumn{2}{|c|}{$\begin{array}{c}\text { Singing } w / \text { head } \\
\text { movements }<\text { singing w/out } \\
\text { head movements } \\
(n=0 / 6)\end{array}$} \\
\hline & $\begin{array}{c}\text { Excited } \\
(3 *)\end{array}$ & $\begin{array}{c}\text { Suppressed } \\
\text { (0) }\end{array}$ & $\begin{array}{l}\text { Excited } \\
\text { (3) }\end{array}$ & $\begin{array}{c}\text { Suppressed } \\
\text { (0) }\end{array}$ & $\begin{array}{c}\text { Excited } \\
(0)\end{array}$ & $\begin{array}{c}\text { Suppressed } \\
\text { (0) }\end{array}$ \\
\hline Singing w/ movements & $0.84 \pm 0.44$ & & $0.49 \pm 0.08$ & & & \\
\hline Singing w/out movements & $0.47 \pm 0.20$ & & $0.32 \pm 0.09$ & & & \\
\hline
\end{tabular}

Neurons are categorized by whether RS during singing that included head movements was greater than (left), equal to (middle), or lower than (right) RS during singing that lacked head movements. Responses are significantly different from quiescence unless otherwise noted (*). Orange lines in Fig. $5 \mathrm{C}$, right, depict head movement responses.

* 1/3 neurons was significantly excited during singing periods that contained head movements but not during singing periods that lacked head movements.

nevertheless showed significant excitation across singing periods (Fig. $5 \mathrm{C}$, right, gray circles; Table 4, excited columns), highlighting the presence of multiple modulating factors during song behavior.

In summary, the singing-modulated activity of most neurons persisted in the absence of head movements (32/36, $89 \%$; Tables 2-4). These results indicate that activity of many Ald neurons during song production may reflect singing-specific movements such as respiratory actions, beak movements, or gular fluttering or non-physical aspects of song production such as auditory-vocal feedback. Interestingly, 12 neurons (33\%) showed differential RS during head movements within singing versus non-singing periods (Fig. 5B,C, right). One interpretation is that these neurons integrate information about head movements and singing behavior, such that changes in firing rate are enhanced specifically during head movements that are performed concurrently with song. Developing associations between head or postural movements and vocal behavior may be a crucial component of learning to produce female-directed song and perform courtship dance movements during singing (Morris, 1954; Balaban, 1997; Williams, 2001; Tomaszycki and AdkinsRegan, 2005). These results raise the possibility that neural activity reflecting "non-singing" movements during song production may be a ubiquitous feature of circuits involved with song learning and control.

\section{Additional sources of Ald neuron modulation: behavioral states}

As indicated above, our goal was to assess the activity of Ald neurons throughout entire sessions of active behaviors. As part of this approach, we devised a novel way of characterizing each recording session by classifying contiguous time periods across each session into one of five different state periods based on the bird's behavior: eating, singing, active-movement, quiet-attentive, or quiescence (Fig. 6A; see Materials and Methods). Eating states were defined as periods when the bird was engaged in eating behavior, including pecking at, hulling, and ingesting seeds; although eating state periods were dominated by eating-related behaviors, other movements such as head movements or hops could also occur. Similarly, singing states included periods when birds were engaged in song production, as well as brief pauses inbetween song bouts during which birds occasionally hopped or pecked. During active-movement states, birds could produce any of the seven movements we scored as well as head and/or postural body movements that were not scored. The remaining two states characterized nonmovement periods: during quiet-attentive states, the bird was alert and could make small head movements but was otherwise not moving; birds made no movements during quiescent states (quiescent states included periods from which baseline intervals were sampled in the scoredmovement analyses above; see Materials and Methods).

For most neurons, firing rates during eating, singing, active-movement, and/or quiet-attentive state periods differed from quiescence (99/109, 91\%; KolmogorovSmirnov test, $p<0.05)$. Few neurons showed changes in firing rate during only one state type; most neurons exhibited firing rate modulations during two or more states (Fig. $6 B$ ). Figure $6 C$ illustrates responsivity of single neurons

Table 4: Mean standardized RS during singing periods with versus without head movements $(n=6$ neurons that showed lower RS during head movements that occurred within singing compared with non-singing periods)

\begin{tabular}{|c|c|c|c|c|c|c|}
\hline & \multicolumn{2}{|c|}{$\begin{array}{c}\text { Singing } w / \text { head } \\
\text { movements }>\text { singing w/out } \\
\text { head movements } \\
(n=3 / 6)\end{array}$} & \multicolumn{2}{|c|}{$\begin{array}{c}\text { Singing } w / \text { head } \\
\text { movements = singing w/out } \\
\text { head movements } \\
(n=1 / 6)\end{array}$} & \multicolumn{2}{|c|}{$\begin{array}{c}\text { Singing } \mathrm{w} / \text { head } \\
\text { movements }<\text { singing w/out } \\
\text { head movements } \\
(n=2 / 6)\end{array}$} \\
\hline Singing w/ movements & 0.35 & $-0.91 \pm 0.70$ & 0.44 & & 0.44 & -2.75 \\
\hline Singing w/out movements & 0.10 & $-0.36 \pm 0.34$ & 0.24 & & 0.74 & -3.88 \\
\hline
\end{tabular}

Neurons are categorized by whether RS during singing that included head movements was greater than (left), equal to (middle), or lower than (right) RS during singing that lacked head movements. All responses are significantly different from quiescence. Gray lines in Fig. $5 \mathrm{C}$, right, depict head movement responses. 
A
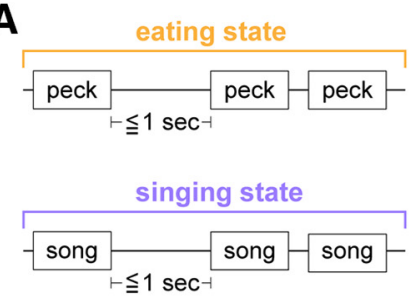

active-movement state

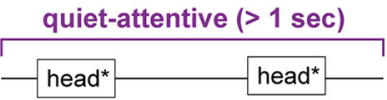

quiescence (>1 sec)
B

number of states responded to

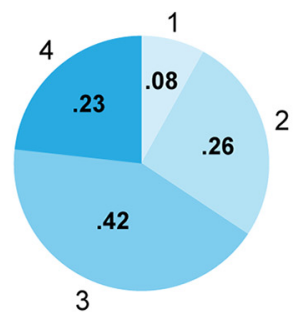

\# state types

* = example of unscored head movement

D active-mvmt eating

singing

quiet-attn

quiescent
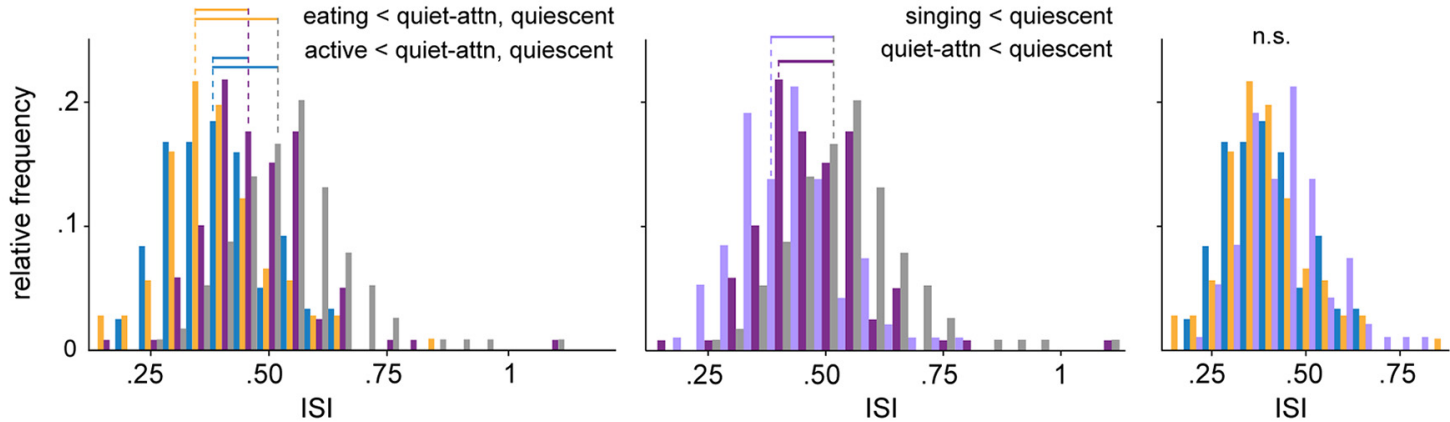

E
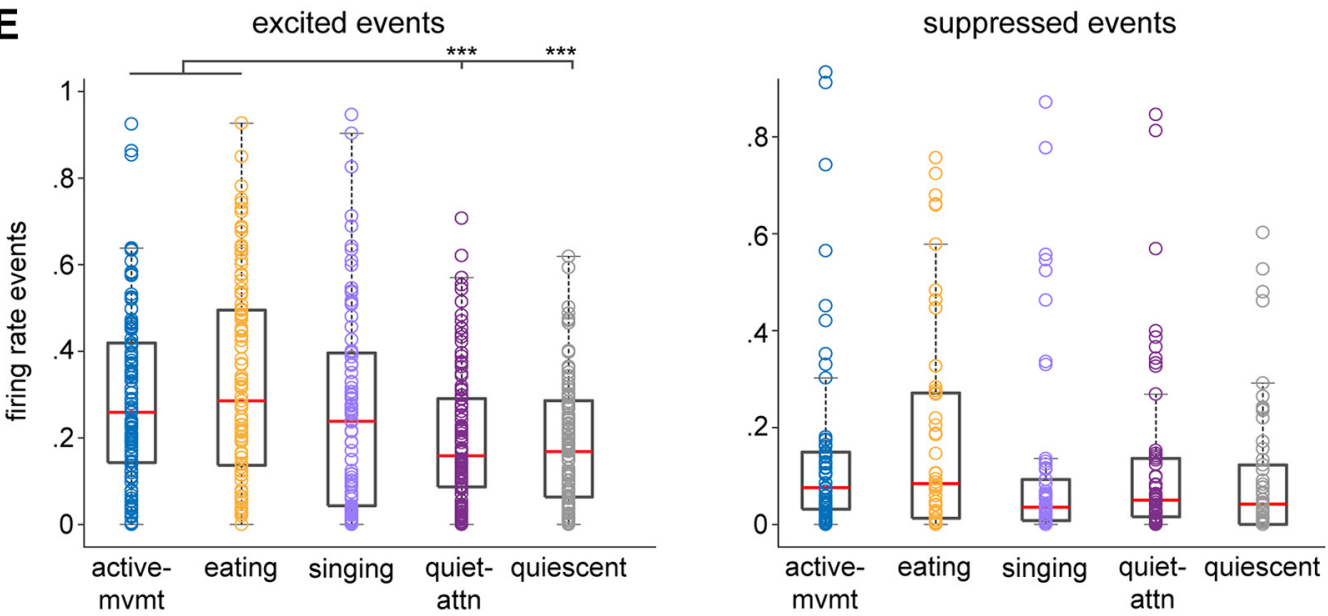

Figure 6. Ald neurons are differentially modulated during different behavioral states. $\boldsymbol{A}$, Schematics of example eating, singing, active-movement, quiet-attentive, and quiescent states. Text boxes represent example scored and unscored (starred) movements that typically occurred within that state type, although other behaviors could also occur (see Materials and Methods). B, Proportions of single Ald neurons that were modulated during different numbers of state types. 8/99 state-responsive neurons were modulated during one state type, 26/99 during two, 42/99 during three, and 23/99 during four states. C. Each row of each chart indicates the states during which each neuron was excited (green), suppressed (dark gray), or not responsive (light gray). Unfilled boxes indicate that no data during that state was recorded for that neuron. Charts are grouped according to colors in $\boldsymbol{B}$, based on the number of 
continued

states during which activity of neurons was modulated. $\boldsymbol{D}$, Histograms comparing distributions of ISIs during active-movement, eating, quiet-attentive, and quiescent states (left); singing, quiet-attentive, and quiescent states (middle); active-movement, eating, and singing states (right). Horizontal lines indicate distributions that had significantly different means; dotted lines indicate means of the respective distributions. $p<0.001$ for all significant differences. $\boldsymbol{E}$, Number of excited (left) and suppressed (right) events that occurred during each state type, normalized by the total duration of each state type in a given recording session. Box-and-whisker plots indicate medians and first and third quartiles; whiskers indicate data points not considered outliers; circles represent data points from individual neurons; $* * * p<0.001$.

during each non-quiescent state type, categorized by the number of states during which each neuron's activity was modulated. Ald neurons could show increased or decreased firing rates during non-quiescent states, and many neurons were excited during one state type and suppressed during another (29/109, 27\%). However, whereas single neurons were equally likely to be suppressed as excited during different discrete scored movements (Fig. 2B), modulation across entire state periods tended to be excitatory: within each state type, the proportion of neurons that were excited was significantly greater than the proportion that were suppressed (binomial test, active-movement and eating states $p<0.0001$, singing and quiet-attentive states $p<0.05$ ), and the overall proportion of excited state responses was greater than suppressed state responses (binomial test, $p<0.0001$ ).

In accord with this pattern of results, ISIs during non-quiescent states were shorter than ISIs during quiescence (Fig. 6D, left, middle; pairwise linear contrasts, BenjaminiHochberg corrected, $p<0.001$ in all cases). In addition, ISIs during active-movement and eating states were shorter than quiet-attentive ISIs ( $p<0.001$ in both cases; Fig. $6 D$, left) and did not differ from singing-state ISIs $(p>0.05$ in both cases; Fig. $6 D$, right). These results indicate greater increases in firing rate during periods of active behavior, particularly for active-movement and eating states.

To capture dynamic changes in activity across state periods, we identified excited and suppressed spiking events during each state type, defined as five or more contiguous 10-ms bins in which the firing rate exceeded the average quiescence firing rate by $\geq 3$ SDs (for excited events) or fell below the average quiescence firing rate by $\leq 1.5$ SDs (for suppressed events). On average, more excited events occurred within active-movement and eating states compared with quiet-attentive and quiescence states (pairwise linear contrasts, Benjamini-Hochberg corrected, $p<0.001$ in all cases; Fig. $6 E$, left). The frequency of excited events during singing states was elevated relative to quiet-attentive and quiescence, but did not differ significantly from any of the other four states (pairwise linear contrasts, Benjamini-Hochberg corrected, $p>0.05$ in all cases; Fig. $6 E$, left). The relatively modest incidence of excited events during singing compared with active-movement and eating states may indicate that firing rate modulation during singing states involves more uniform increases in tonic spike rate. The number of suppressed events did not differ between state types (pairwise linear contrasts, Benjamini-Hochberg corrected, $p>0.05$ in all cases; Fig. $6 E$, right). Taken together, these results indicate an increase in firing rate during non-quiescent states, with a greater degree of excitatory modulation during active-movement, eating and singing states expressed as shorter ISIs as well as an increase in discrete high-firing periods during activemovement and eating states.

\section{How does activity during scored movements contribute to behavioral states?}

Because eating and singing state periods were characterized primarily by one type of scored movement (eatingrelated pecks and singing bouts, respectively; Fig. 7A), we wondered whether excitation during each of these states was restricted to neurons that were excited during the corresponding scored movements. If state responsivity of single neurons can be attributed to their movement responsivity, then neurons excited during eating states should also be excited during eating-related pecks, which accounted for $99 \%$ of scored movements during eating. Similarly, neurons excited during singing state periods should also be excited during song bouts. In contrast, if behavioral states include other sources of modulation, then we might expect to find a more diverse pattern of movement-related responsivity among eating-state-excited and singing-state-excited neurons; this latter outcome would be consistent with the heterogeneous pattern of movement responsivity described above.

Figure $7 B$, left, illustrates the peck responsivity of 48 neurons that were excited during eating states, grouped by their response during eating-related pecks. A majority of neurons that were excited during eating states were also excited during discrete peck movements (31/48, $65 \%)$. However, the remaining $35 \%$ of eating-state-excited neurons were suppressed or unresponsive during eating-related pecks, indicating that the heightened firing rate of these neurons during eating states did not relate to pecking behavior. Increased firing rates in these latter cells may be related to unscored factors during eating such as head movements or hulling behavior, or external sensory inputs. Likewise, most of the neurons that were excited during singing state periods were excited during song bouts $(26 / 37,70 \%)$, but the remaining $30 \%$ were suppressed or unresponsive during song bouts (Fig. 7C, left). Thus, excitation across singing states in these latter neurons presumably reflects activity during unscored factors that occur in-between song bouts.

Consistent with the fact that most neurons responded during multiple movements and states (Figs. 2, 6), neurons excited during eating and singing state periods were also responsive during a variety of scored movements that did not occur within these states. For example, many eating-state-excited neurons also responded during song 
A

hop
peck
preening
beak inter
fluff-up
scratch
stretch
song bout

relative proportions of scored behaviors that occurred in each state

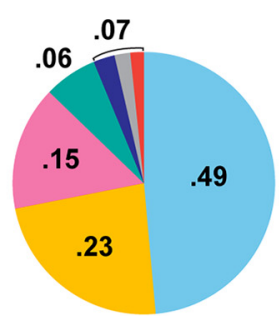

active-movement

$$
n=4367
$$

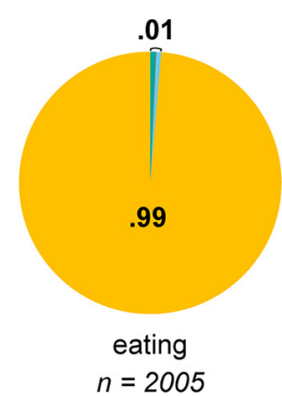

$n=2005$

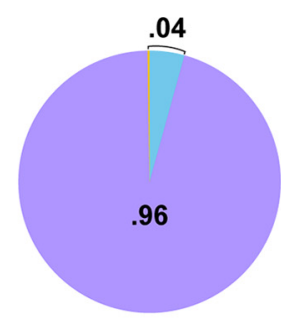

singing

$n=1628$
B eating state-excited cells

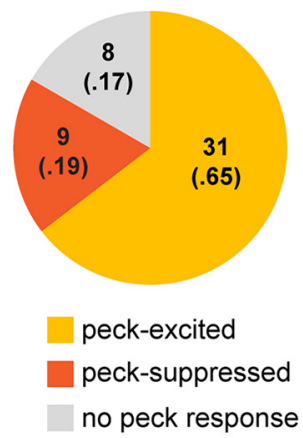

C

singing state-excited cells

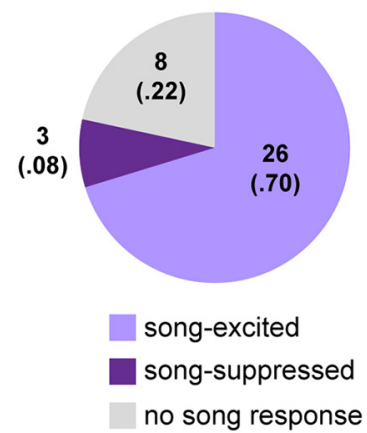

eating state-excited, peck-excited

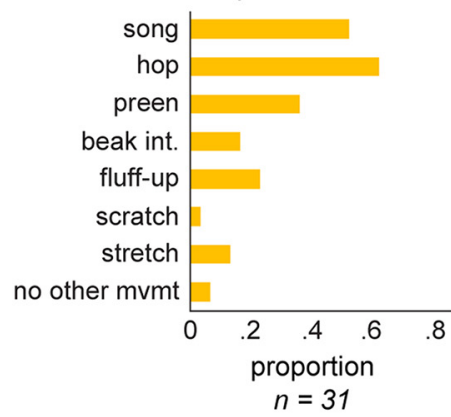

singing state-excited, song-excited

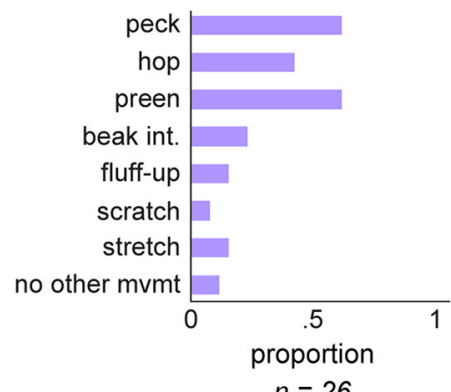

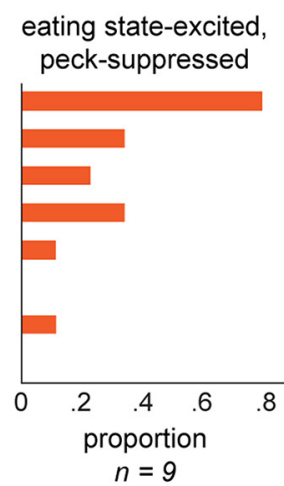

singing state-excited, song-suppressed

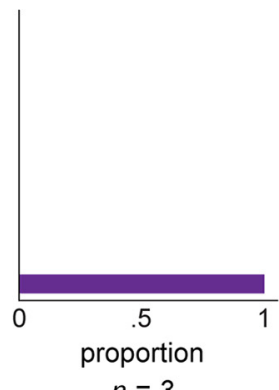

eating state-excited no peck response

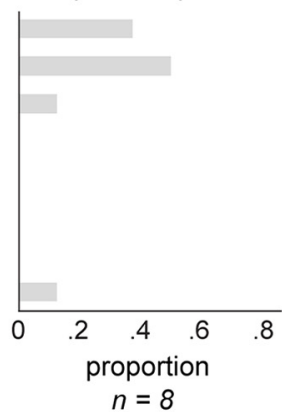

singing state-excited, no song response

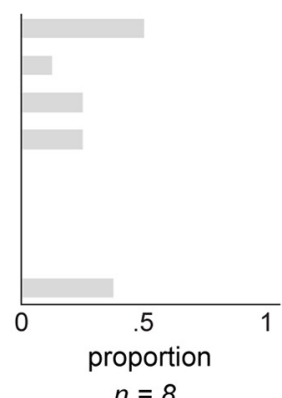

Figure 7. Movement-excited Ald neurons are modulated by scored and unscored factors during different state periods. $\boldsymbol{A}$, Relative proportions of scored behaviors that occurred during each state type. Proportion totals include all occurrences of each of the seven scored movements as well as all song bouts. $\boldsymbol{B}$, left, Peck responsivity of 48 neurons that were significantly excited during eating state periods. Right, Proportions of eating-excited neurons that were responsive during scored behaviors, categorized by peck responsivity: eating-excited neurons that were peck-excited (left), peck-suppressed (middle), and unresponsive during pecks (right). "No other movement" represents neurons that did not respond during any other scored behavior. C, left, Song-bout responsivity of 37 neurons that were significantly excited during singing state periods. Right, Proportions of singing state-excited neurons that were responsive during scored movements, categorized by song responsivity: singing state-excited neurons that were song-excited (left), song-suppressed (middle), and unresponsive during song bouts (right). "No other movement" represents neurons that did not respond during any other scored behavior. Italicized numbers indicate number of neurons within each song-responsivity grouping.

bouts, preening, and fluff-ups, even though these behaviors never occurred within eating states (Fig. $7 A, B$, right). Similarly, most neurons excited during singing states were also responsive during scored movements that did not occur within singing states, such as preening, beak interactions, and fluff-ups (Fig. $7 A, C$, right).

These results highlight the complexity of information that Ald neurons are processing. In many instances, single neurons were excited during a given behavioral state, but not during the scored movement that characterized that state. For example, some neurons were excited during eating states but suppressed during discrete eating-related pecks, indicating that the overall excitation seen across eating states was due in part to some other (non-pecking) influence. In addition, many neurons that showed excitation during a behavioral state were also responsive during multiple scored movements that were unrelated to that state. For example, an eating-state-excited neuron could also respond 
during movements that occurred outside of eating states, such as preening or fluff-ups. Thus, single neurons could demonstrate increased firing rates during state periods that were unrelated to scored movements, while also exhibiting modulation during specific movements that occur outside of that state type.

In addition, while the majority of neurons we recorded were responsive during one or more scored movements (Fig. 2), 18 out of 119 cells (15\%) were not significantly modulated during any scored movement. However, firing rates of most of these "movement-unresponsive" neurons were modulated during at least one state type compared with quiescence (15/18, 83\%; Fig. 8). This result suggests that some of the neurons unresponsive during scored movements nonetheless exhibit firing rate changes related to unscored factors as the juvenile is actively behaving.

\section{Discussion}

\section{Heterogeneous activity within Ald reflects multidimensional tuning}

We found that most Ald neurons were selective for single movements or for different combinations of movements. Neurons responsive during different movements frequently demonstrated excitation during one movement and suppression during another. Moreover, individual responses could include transient excitation at movement onset and/or offset as well as suppression of average firing rate during the movement itself, or vice versa. The diversity of neural responses in Ald is strikingly similar to the response profile of neurons in macaque motor cortex, where single neurons demonstrate heterogeneous, multiphasic temporal patterns of activity across reaching movements (Churchland and Shenoy, 2007). Such complex responses may result from multiple inputs relating to different movements or aspects of movements onto single Ald neurons, as well as local transformation of afferent inputs. Ald includes a local inhibitory network, evidenced by the fact that blocking GABA-A receptors in Ald of anesthetized zebra finches elicits increased spontaneous firing rates, and parvalbumin expression is higher in Ald compared with surrounding motor cortex (Mello et al., 2019; Yuan and Bottjer, 2019). Similarly, mammalian motor cortex contains a substantial population of inhibitory interneurons, which have been implicated in both regulating plasticity during motor skill learning and coordinating activity across motor cortex during behavior (Jacobs and Donoghue, 1991; Hess and Donoghue, 1994; Hess et al., 1996; Markram et al., 2004; Stagg et al., 2011; Donato et al., 2013; Chen et al., 2015; Kida et al., 2016; Adler et al., 2019). Blocking inhibition may unmask latent excitatory connections between spatially distant motor cortical neurons, providing a mechanism by which dynamic modulation of inhibition could flexibly reorganize connectivity and coordinate population activity across motor cortex (Jacobs and Donoghue, 1991; Spiro et al., 1999; Schneider et al., 2002; Capaday, 2004). Ald receives topographic input from parallel circuits that process auditory, visual, and somatosensory information, so a similar mechanism to link different neuronal subpopulations within

\section{A neurons with no significant response during scored movements}

number of states responded to

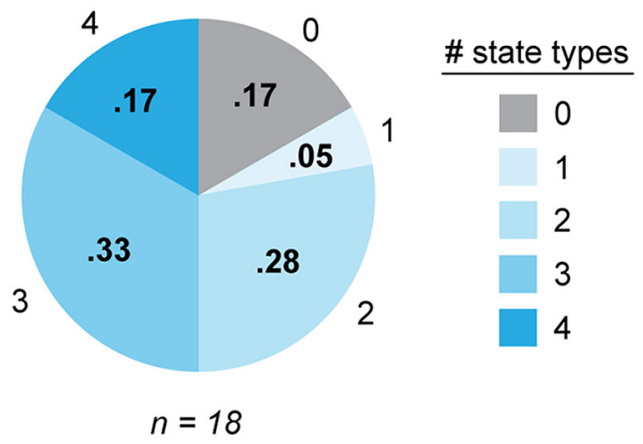

B

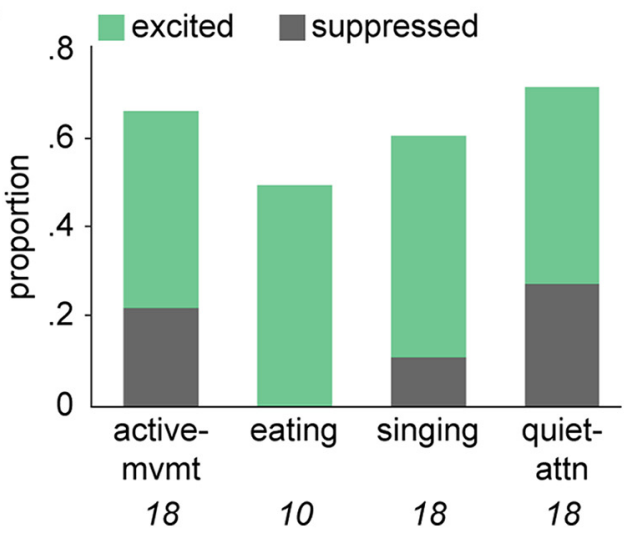

Figure 8. Ald neurons that lack responsivity during scored movements are nonetheless modulated during different states. $\boldsymbol{A}$, Proportions of non-responsive Ald neurons that were modulated during different numbers of state types. 3/18 neurons were not modulated during any states; $1 / 18$ was modulated during one state type, 5/18 during two, 6/18 during three, and $3 / 18$ during four state types. $\boldsymbol{B}$, Proportions of non-responsive neurons that were significantly excited (green) or suppressed (dark gray) during each state type. Italicized numbers indicate number of non-responsive neurons recorded during each state type.

Ald would be advantageous for facilitating sensorimotor integration across modalities (Zeier and Karten, 1971; Bottjer et al., 2000; Paterson and Bottjer, 2017).

The heterogeneous response profile of motor cortical neurons across taxa raises interesting questions about what factors contribute to the tuning of these neurons. Modulation of motor cortical activity has been associated with a variety of behavioral parameters in arm-reaching tasks, including movement direction, speed, trajectory, limb position, and joint angle (Evarts, 1968; Cheney and Fetz, 1980; Georgopoulos et al., 1982, 1988; Schwartz et al., 1988; Fu et al., 1993; Schwartz and Moran, 2000; Reina et al., 2001; Paninski et al., 2004; Churchland and Shenoy, 2007; Hatsopoulos et al., 2007). Increasing evidence indicates that multiple parameters can be reflected in the activity of single neurons, suggesting that integrated multimodal tuning may be a fundamental feature of 
motor cortical activity. For instance, recordings from macaques during unrestrained arm movements showed that parameters such as movement direction or end position of the limb could account for only a portion of spiking patterns in single motor cortical neurons, indicating that individual neurons may be tuned in a multidimensional space and that testing neural activity relative to any single parameter may account only partially for multidimensional tuning profiles (Ashe and Georgopoulos, 1994; Fu et al., 1995; Moran and Schwartz, 1999; Aflalo and Graziano, 2006, 2007). Likewise, we found that single Ald neurons could be modulated both during diverse individual movements and during behavioral state periods that did not include those movements, indicating that single neurons were modulated by multiple factors.

Given convergent input from a diverse array of processing streams, the tuning profiles of neurons in both avian and mammalian motor cortex are not limited to motor responsivity. For instance, neurons in RA, which lies adjacent to Ald in songbird motor cortex, drive vocal motor output in singing birds and demonstrate robust responses to playback of song stimuli in anesthetized or sleeping birds (Nottebohm et al., 1976; Doupe and Konishi, 1991; Vicario and Yohay, 1993; Wild, 1993; Yu and Margoliash, 1996; Dave et al., 1998; Dave and Margoliash, 2000; Leonardo and Fee, 2005; Kojima and Doupe, 2007; Sober et al., 2008; Yuan and Bottjer, 2019). Recordings from macaque motor cortex have likewise demonstrated sensitivity to non-motor stimuli: for instance, in visually-guided target-reaching paradigms, some motor cortical neurons exhibit selective activity related to the visual target, regardless of the limb trajectory used to reach that target (Tanji and Evarts, 1976; Evarts and Fromm, 1977; Murata et al., 1997; Shen and Alexander, 1997). The activity of Ald neurons may be similarly modulated by integration of various factors to produce heterogeneous responses with complex temporal patterning during diverse movements. In support of this idea, we found neurons whose activity was modulated across behavioral state periods but not during any scored movements (Fig. 8). Although this activity could be related to head movements, which we did not score comprehensively, another possibility is that activity in these neurons relates to non-motor factors such as visual processing, attention, or arousal (Knudsen et al., 1995; Rauske et al., 2003; Cardin and Schmidt, 2004; Winkowski and Knudsen, 2007; Fernández et al., 2020).

\section{Multimodal integration provides behavioral context for voluntary movements}

We found that most Ald neurons demonstrated altered response strength during movements relative to quiescence; under the conditions of our recordings, firing rate modulations occurred most often during pecks, preening, and/or hops. It is difficult to know whether movement-related activity in Ald neurons is pre-motor, modulatory, or reflective of movement feedback or external sensory inputs. Ald neurons project to several targets, including the striatum, a dorsal thalamic zone, the lateral hypothalamus, a thalamic nucleus that relays to cerebellum [medial spiriform nucleus (SpM)], deep layers of the tectum, broad areas of the pontine and midbrain reticular formation, and VTA (Fig. 1A; Bottjer et al., 2000). The medial pontine reticular formation contains premotor neurons that contribute to neck and locomotive movements in other avian species (Steeves et al., 1987; Valenzuela et al., 1990; Dubbeldam, 1998; Wild and Krützfeldt, 2012); peck, preening, and hop-related activity may in part reflect these projections to premotor centers. Previous studies have found evidence of increased expression of the immediate early gene egr-1 in Ald specifically during hopping behavior (Feenders et al., 2008), which could indicate motor-related activity.

While these studies may indicate a role for Ald in motor execution, other lines of evidence suggest that movement-related activity in many Ald neurons is unlikely to reflect direct motor drive of peck, preening, and/or hopping behavior per se. Importantly, lesions of Ald in juvenile birds do not disrupt song output or induce noticeable motor deficits, suggesting that Ald neurons are not driving voluntary pecking or hopping movements (Bottjer and Altenau, 2010; see Mandelblat-Cerf et al., 2014; Materials and Methods). Moreover, the results presented here demonstrate that single Ald neurons do not respond consistently during one particular type of movement. For instance, we found a substantial population of peck-responsive neurons that modulated their firing rate during pecks when the bird was eating but not when the bird pecked at other objects around the cage (or vice versa), even though pecking movements in these different contexts would recruit many of the same muscle groups. Furthermore, behavioral and functional experiments across avian species have implicated intermediate arcopallium in highly integrative, complex behaviors that extend beyond pure motor control, including ingestive behaviors, working memory processing, fear conditioning, and vocal learning (Lowndes and Davies, 1994; Lowndes et al., 1994; Knudsen and Knudsen, 1996; Aoki et al., 2006; Campanella et al., 2009; Saint-Dizier et al., 2009; Bottjer and Altenau, 2010; Achiro et al., 2017). One such example is a region comprising caudal arcopallium and nidopallium, which partially overlaps with Ald and exhibited increased 2deoxyglucose uptake when adult male zebra finches participated in their first courtship experience following several weeks of isolation from females; the amount of glucose consumption in this region correlated positively with isolation time but not with amount of movement activity (Bischof and Herrmann, 1986).

Rather than generically driving motor behavior per se, an important function of motor cortical circuitry is incorporating sensory information to appropriately direct motor output based on an animal's environment and/or goals; this sensorimotor integration is necessary for voluntary movements such as goal-directed motor behaviors (e.g., object-directed grasping) as well as adaptive movements based on environmental perturbations. Avian and mammalian motor cortices receive multimodal inputs and target brainstem regions, making them ideally situated to carry out sensorimotor integration during voluntary behaviors. In macaques, neurons in motor cortical areas 
demonstrate a selective response when grasping at a particular object and corresponding visual selectivity for the same object when the monkey fixates on the object without grasping; inactivation of the same motor cortical region resulted in grasping deficits because of disrupted preparatory hand shaping that was inappropriate for the target object, suggesting a specific impairment in visuomotor transformations for targeted grasping rather than a gross motor impairment of hand movements (Murata et al., 1997; Fogassi et al., 2001; Rizzolatti and Luppino, 2001). Some Ald neurons may serve a similar function in integrating sensory information to provide appropriate context for motor output. For example, the "eating-peck" responses observed here could represent an integrated response when the visual stimulus of seed is present as the bird pecks; these neurons could link visual information about seed with somatosensory information to contribute specifically to food-directed pecking behavior. In contrast, neurons that showed excitation during pecks associated with non-eating behaviors may process diverse inputs in the context of object exploration. Notably, some Ald neurons could be involved in sensory-motor integration and also play a role in motor execution; likewise, other Ald neurons could provide appropriate context for voluntary behaviors without directly driving motor actions. For instance, rather than directly linking sensory information to premotor centers, some neurons could instead feed multimodal information back into ascending reticular or tectal pathways to contribute to goal-directed behaviors, or differentially ascribe value to environmental cues depending on the animal's current state or needs (Burgess et al., 2018).

\section{Ald is uniquely situated to mediate learning and performance of both vocal and non-vocal elements of song behavior}

Although motor cortex is generally well situated to integrate multimodal information related to a variety of goaldirected movements, Ald's unique connections suggest it may occupy a specific role in vocal learning and behavior. LMAN-CORE neurons that drive vocal motor output in juvenile birds make robust collateral projections into Ald at 20-35 dph that substantially decline by $45 \mathrm{dph}$ (MillerSims and Bottjer, 2012; J. H. Chung and S. W. Bottjer, unpublished observations). While our dataset did not include ages young enough to test the functional role of this connection between LMAN-CORE and Ald, information from this developmentally regulated projection may play a critical role during the earliest stages of vocal practice and influence patterns of connectivity within Ald that contribute to sensorimotor processing during subsequent learning. In addition, Ald projects to higher-order thalamic nuclei that are linked to vocal learning, the dorsolateral nucleus of the medial thalamus (DLM) and the dorsomedial nucleus of the posterior thalamus (DMP; Fig. 1A; Bottjer et al., 2000). DLM is required for normal song behavior and projects to LMAN-SHELL, whereas DMP projects to the medial magnocellular nucleus of anterior nidopallium (MMAN); both LMAN and MMAN are required for development of an accurate imitation of tutor song (Bottjer et al.,
1984; Foster et al., 1997; Vates et al., 1997; Foster and Bottjer, 2001; Aronov et al., 2008; Goldberg and Fee, 2011; Chen et al., 2014). Ald also projects to lateral hypothalamus, striatum, and the area of dopaminergic neurons in VTA that projects to a nucleus in avian basal ganglia necessary for song learning (Fig. 1A; Bottjer et al., 2000); these limbic-related projections further suggest that Ald neurons are well situated to contribute to vocal learning and behavior.

One hypothesis to integrate these unique connections with the multimodal integrative function of motor cortex is that some Ald neurons may be involved in mediating learning and performance of movements in the context of song behavior. Song production in zebra finches is a courtship behavior during which males vocalize while performing a dance-like sequence of hopping movements oriented toward a female (Morris, 1954; Williams, 2001; Cooper and Goller, 2004; Dalziell et al., 2013; Ota et al., 2015; Ullrich et al., 2016); the temporal patterning of dance movements during song production is significantly correlated between father-son pairs of zebra finches, suggesting that non-vocal behaviors that accompany singing may be learned as well (Williams, 2001). Establishing a social context for courtship behavior likely involves sensory cues. For instance, adult males can use visual cues to select between two female birds shown in a silent video feed, but the addition of auditory cues induces stronger courtship responses (Galoch and Bischof, 2006, 2007). Ald neurons are ideally positioned to integrate environmental context cues when females are present to guide learning and performance of movements that accompany singing.

In this framework, the relatively high proportions of peck, preening, and hopping-related responses observed here may reflect the fact that beak movements and hopping are important components of courtship behavior. In quail-chick chimeras, chicks that received transplants of lower brainstem somites from quails retained chick-like call structures but adopted quail-like patterns of head movements specifically during vocalizations; similar head movements made outside of vocalization periods were not affected, indicating the presence of specialized circuitry that mediates movements in the context of vocal behavior (Balaban, 1997). Circuitry processing non-vocal elements of singing in zebra finches may be similarly specialized; our results suggest that investigating how activity patterns during singing correspond to movements of different peripheral targets would benefit hypotheses for mechanisms of song production: for instance, we found a subpopulation of Ald neurons that exhibited differential response strength during head movements that occurred during singing versus nonsinging periods. These responses may indicate integration between non-vocal and vocal elements of song behavior, such that neural activity is enhanced specifically during head movements that accompany singing. This hypothesis raises an interesting prediction: although Ald lesions in juvenile birds do not induce any gross motor deficits, it is possible that hopping and/or head movements performed during song production would be disrupted. Such a result would be consistent with previous studies in which c-Fos expression in Ald was increased after adult male zebra finches performed 
non-singing courtship behaviors directed toward a live female (Kimpo and Doupe, 1997). Involvement of Ald in courtship-related movements would draw an interesting parallel to mammalian studies that have suggested that motor cortex is parceled into "action zones" that each process information for different ethologically relevant categories of movement (Graziano, 2006; Graziano and Aflalo, 2007). For instance, stimulation of one region of macaque motor cortex results in the animal closing its hand in a grip while bringing it to its mouth and opening its mouth, as if eating an object, while stimulation of another region results in the monkey raising its arm and turning its head sharply to one side as if in defense (Graziano et al., 2002). In this context, adjacent motor cortical regions $\mathrm{RA}$ and Ald could serve as an action zone that mediates the vocal and non-vocal movements that comprise song behavior.

While brainstem projections may mediate sensorimotor processing during vocal motor performance, the thalamic and midbrain projections of Ald that give rise to recurrent feedback loops through cortico-basal ganglia circuitry may integrate multimodal information to facilitate song learning. Although Ald does not drive song output (Bottjer et al., 2000; Bottjer and Altenau, 2010), we found a substantial population of singing-responsive neurons. For a large proportion of these neurons, the changes in firing rate during singing could not be attributed to any of the movements that we scored (including head movements). These firing rate modulations may instead be related to singing-specific movements such as beak movements or gular fluttering, or sensory activity such as auditory or proprioceptive feedback (Goller et al., 2004; Ohms et al., 2010; Bottjer and To, 2012; Riede et al., 2013). Alternatively, singing-related activity could reflect active evaluations of the juvenile's vocal behavior during sensorimotor practice; iterative evaluations between self-generated output and the goal tutor song are essential for guiding accurate refinement of the juvenile's song, and evidence of neural activity processing these comparisons has been reported in LMAN-SHELL, which projects directly to Ald (Achiro et al., 2017). Importantly, successful song learning also requires multiple factors beyond simply matching vocal output to an auditory goal. For instance, vocal learning in juvenile zebra finches that are tutored with only passive playback of the tutor song is severely impaired, whereas pairing auditory tutoring with a visual model of an adult zebra finch enhances learning (Derégnaucourt et al., 2013; Ljubičić et al., 2016). Moreover, visual cues provided during singing, such as wing strokes or fluff-ups from adult females, provide feedback that can influence juvenile vocal learning (West and King, 1988; Morrison and Nottebohm, 1993; King et al., 2005; Carouso-Peck and Goldstein, 2019). Multimodal inputs from $\mathrm{dNCL}$ and singing-related inputs from LMAN may converge in Ald, integrating important nonvocal and vocal elements of courtship song behavior that must be learned during a sensitive period of development.

\section{References}

Achiro JM, Bottjer SW (2013) Neural representation of a target auditory memory in a cortico-basal ganglia pathway. J Neurosci 33:14475-14488.

Achiro JM, Shen J, Bottjer SW (2017) Neural activity in cortico-basal ganglia circuits of juvenile songbirds encodes performance during goal-directed learning. Elife 6:e26973.
Adler A, Zhao R, Shin ME, Yasuda R, Gan WB (2019) Somatostatin-expressing interneurons enable and maintain learning-dependent sequential activation of pyramidal neurons. Neuron 102:202-216.e7.

Aflalo TN, Graziano MSA (2006) Partial tuning of motor cortex neurons to final posture in a free-moving paradigm. Proc Natl Acad Sci USA 103:2909-2914.

Aflalo TN, Graziano MSA (2007) Relationship between unconstrained arm movements and single-neuron firing in the macaque motor cortex. J Neurosci 27:2760-2780.

Aoki N, Csillag A, Matsushima T (2006) Localized lesions of arcopallium intermedium of the lateral forebrain caused a handling-cost aversion in the domestic chick performing a binary choice task. Eur J Neurosci 24:2314-2326.

Aronov D, Andalman AS, Fee MS (2008) A specialized forebrain circuit for vocal babbling in the juvenile songbird. Science 320:630634.

Ashe J, Georgopoulos AP (1994) Movement parameters and neural activity in motor cortex and area 5. Cereb Cortex 4:590-600.

Balaban E (1997) Changes in multiple brain regions underlie species differences in a complex, congenital behavior. Proc Natl Acad Sci USA 94:2001-2006.

Benjamini Y, Hochberg Y (1995) Controlling the false discovery rate: a practical and powerful approach to multiple testing. J R Stat Soc 57:289-300.

Bischof HJ, Herrmann K (1986) Arousal enhances [14C]2-deoxyglucose uptake in four forebrain areas of the zebra finch. Behav Brain Res 21:215-221.

Böhner J (1983) Song learning in the zebra finch (Taeniopygia guttata): selectivity in the choice of a tutor and accuracy of song copies. Anim Behav 31:231-237.

Böhner J (1990) Early acquisition of song in the zebra finch, Taeniopygia guttata. Anim Behav 39:369-374.

Bottjer SW, Altenau B (2010) Parallel pathways for vocal learning in basal ganglia of songbirds. Nat Neurosci 13:153-155.

Bottjer SW, To M (2012) Afferents from vocal motor and respiratory effectors are recruited during vocal production in juvenile songbirds. J Neurosci 32:10895-10906.

Bottjer SW, Miesner EA, Arnold AP (1984) Forebrain lesions disrupt development but not maintenance of song in passerine birds. Science 224:901-903.

Bottjer SW, Brady JD, Cribbs B (2000) Connections of a motor cortical region in zebra finches: relation to pathways for vocal learning. J Comp Neurol 420:244-260.

Burgess CR, Livneh Y, Ramesh RN, Andermann ML (2018) Gating of visual processing by physiological need. Curr Opin Neurobiol 49:16-23.

Campanella LCA, da Silva AA, Gellert DS, Parreira C, Ramos MC, Paschoalini MA, Marino-Neto J (2009) Tonic serotonergic control of ingestive behaviours in the pigeon (Columba livia): the role of the arcopallium. Behav Brain Res 205:396-405.

Capaday C (2004) The integrated nature of motor cortical function. Neuroscientist 10:207-220.

Cardin JA, Schmidt MF (2004) Auditory responses in multiple sensorimotor song system nuclei are co-modulated by behavioral state. J Neurophysiol 91:2148-2163.

Carouso-Peck S, Goldstein MH (2019) Female social feedback reveals non-imitative mechanisms of vocal learning in zebra finches. Curr Biol 29:631-636.e3.

Chen JR, Stepanek L, Doupe AJ (2014) Differential contributions of basal ganglia and thalamus to song initiation, tempo, and structure. J Neurophysiol 111:248-257.

Chen SX, Kim AN, Peters AJ, Komiyama T (2015) Subtype-specific plasticity of inhibitory circuits in motor cortex during motor learning. Nat Neurosci 18:1109-1115.

Chen Y, Matheson LE, Sakata JT (2016) Mechanisms underlying the social enhancement of vocal learning in songbirds. Proc Natl Acad Sci USA 113:6641-6646.

Cheney PD, Fetz EE (1980) Functional classes of primate corticomotoneuronal cells and their relation to active force. J Neurophysiol 44:773-791. 
Churchland MM, Shenoy KV (2007) Temporal complexity and heterogeneity of single-neuron activity in premotor and motor cortex. $\mathrm{J}$ Neurophysiol 97:4235-4257.

Cooper BG, Goller F (2004) Multimodal signals: enhancement and constraint of song motor patterns by visual display. Science 303:544-546.

Dalziell AH, Peters RA, Cockburn A, Dorland AD, Maisey AC, Magrath RD (2013) Dance choreography is coordinated with song repertoire in a complex avian display. Curr Biol 23:1132-1135.

Darling WG, Pizzimenti MA, Morecraft RJ (2011) Functional recovery following motor cortex lesions in non-human primates: experimental implications for human stroke patients. J Integr Neurosci 10:353-384

Dave AS, Margoliash D (2000) Song replay during sleep and computational rules for sensorimotor vocal learning. Science 290:812816.

Dave AS, Yu AC, Margoliash D (1998) Behavioral state modulation of auditory activity in a vocal motor system. Science 282:2250-2254.

Derégnaucourt S, Poirier C, Kant A, Van der Linden A, Van der Gahr M (2013) Comparisons of different methods to train a young zebra finch (Taeniopygia guttata) to learn a song. J Physiol Paris 107:210-218.

Donato F, Rompani SB, Caroni P (2013) Parvalbumin-expressing basket-cell network plasticity induced by experience regulates adult learning. Nature 504:272-276.

Doupe AJ, Konishi M (1991) Song-selective auditory circuits in the vocal control system of the zebra finch. Proc Natl Acad Sci USA 88:11339-11343.

Dubbeldam JL (1998) The neural substrate for "learned" and "nonlearned" activities in birds: a discussion of the organization of bulbar reticular premotor systems with side-lights on the mammalian situation. Acta Anat (Basel) 163:157-172.

Eales LA (1985) Song learning in zebra finches: some effects of song model availability on what is learnt and when. Anim Behav 33:1293-1300.

Eales LA (1989) The influences of visual and vocal interaction on song learning in Zebra finches. Anim Behav 37:507-508.

Ebbesen CL, Doron G, Lenschow C, Brecht M (2017) Vibrissa motor cortex activity suppresses contralateral whisking behavior. Nat Neurosci 20:82-89.

Evarts EV (1968) Relation of pyramidal tract activity to force exerted during voluntary movement. J Neurophysiol 31:14-27.

Evarts EV, Fromm C (1977) Sensory responses in motor cortex neurons during precise motor control. Neurosci Lett 5:267-272.

Feenders G, Liedvogel M, Rivas M, Zapka M, Horita H, Hara E, Wada $\mathrm{K}$, Mouritsen H, Jarvis ED (2008) Molecular mapping of movement-associated areas in the avian brain: a motor theory for vocal learning origin. PLoS One 3:e1768.

Ferezou I, Haiss F, Gentet LJ, Aronoff R, Weber B, Petersen CCH (2007) Spatiotemporal dynamics of cortical sensorimotor integration in behaving mice. Neuron 56:907-923.

Fernández M, Morales C, Durán E, Fernández-Colleman S, Sentis E, Mpodozis J, Karten HJ, Marín GJ (2020) Parallel organization of the avian sensorimotor arcopallium: tectofugal visual pathway in the pigeon (Columba livia). J Comp Neurol 528:597-623.

Fogassi L, Gallese V, Buccino G, Craighero L, Fadiga L, Rizzolatti G (2001) Cortical mechanism for the visual guidance of hand grasping movements in the monkey: a reversible inactivation study. Brain 124:571-586.

Foster EF, Bottjer SW (2001) Lesions of a telencephalic nucleus in male zebra finches: influences on vocal behavior in juveniles and adults. J Neurobiol 46:142-165.

Foster EF, Mehta RP, Bottjer SW (1997) Axonal connections of the medial magnocellular nucleus of the anterior neostriatum in zebra finches. J Comp Neurol 382:364-381.

Fu QG, Suarez JI, Ebner TJ (1993) Neuronal specification of direction and distance during reaching movements in the superior precentral premotor area and primary motor cortex of monkeys. J Neurophysiol 70:2097-2116.
Fu QG, Flament D, Coltz JD, Ebner TJ (1995) Temporal encoding of movement kinematics in the discharge of primate primary motor and premotor neurons. J Neurophysiol 73:836-854.

Gale SD, Person AL, Perkel ADJ (2008) A novel basal ganglia pathway forms a loop linking a vocal learning circuit with its dopaminergic input. J Comp Neurol 508:824-839.

Galoch Z, Bischof HJ (2006) Zebra finches actively choose between live images of conspecifics. Ornithol Sci 5:57-64.

Galoch Z, Bischof HJ (2007) Behavioural responses to video playbacks by zebra finch males. Behav Processes 74:21-26.

Georgopoulos AP, Kalaska JF, Caminiti R, Massey JT (1982) On the relations between the direction of two-dimensional arm movements and cell discharge in primate motor cortex. J Neurosci 2:1527-1537.

Georgopoulos AP, Kettner RE, Schwartz AB (1988) Primate motor cortex and free arm movements to visual targets in three-dimensional space. II. Coding of the direction of movement by a neuronal population. J Neurosci 8:2928-2937.

Goldberg JH, Fee MS (2011) Vocal babbling in songbirds requires the basal ganglia-recipient motor thalamus but not the basal ganglia. J Neurophysiol 105:2729-2739.

Goller F, Mallinckrodt MJ, Torti SD (2004) Beak gape dynamics during song in the zebra finch. J Neurobiol 59:289-303.

Graziano M (2006) The organization of behavioral repertoire in motor cortex. Annu Rev Neurosci 29:105-134.

Graziano MSA, Aflalo TN (2007) Mapping behavioral repertoire onto the cortex. Neuron 56:239-251.

Graziano MS, Taylor CS, Moore T (2002) Complex movements evoked by microstimulation of precentral cortex. Neuron 34:841851.

Guo JZ, Graves AR, Guo WW, Zheng J, Lee A, Rodríguez-Gonzá Lez J, Li N, Macklin JJ, Phillips JW, Mensh BD, Branson K, Hantman AW (2015) Cortex commands the performance of skilled movement. Elife 4:e10774.

Hatsopoulos NG, Xu Q, Amit Y (2007) Encoding of movement fragments in the motor cortex. J Neurosci 27:5105-5114.

Hess G, Donoghue JP (1994) Long-term potentiation of horizontal connections provides a mechanism to reorganize cortical motor maps. J Neurophysiol 71:2543-2547.

Hess G, Aizenman CD, Donoghue JP (1996) Conditions for the induction of long-term potentiation in layer II/III horizontal connections of the rat motor cortex. J Neurophysiol 75:1765-1778.

Hwang EJ, Dahlen JE, Hu YY, Aguilar K, Yu B, Mukundan M, Mitani A, Komiyama T (2019) Disengagement of motor cortex from movement control during long-term learning. Sci Adv 5:eaay0001.

Immelmann K (1969) Song development in the zebra finch and other estrildid finches. In: Bird vocalizations (Hinde RA, ed), pp 61-74. Cambridge: Cambridge University Press.

Jacobs K, Donoghue J (1991) Reshaping the cortical motor map by unmasking latent intracortical connections. Science 251:944-947.

Karten HJ (2013) Neocortical evolution: neuronal circuits arise independently of lamination. Curr Biol 23:R12-R15.

Kawai R, Markman T, Poddar R, Ko R, Fantana AL, Dhawale AK, Kampff AR, Ölveczky BP (2015) Motor cortex is required for learning but not for executing a motor skill. Neuron 86:800-812.

Kelley DB, Nottebohm F (1979) Projections of a telencephalic auditory nucleus-field L-in the canary. J Comp Neurol 183:455-469.

Kida $\mathrm{H}$, Tsuda $\mathrm{Y}$, Ito $\mathrm{N}$, Yamamoto $\mathrm{Y}$, Owada $\mathrm{Y}$, Kamiya $\mathrm{Y}$, Mitsushima D (2016) Motor training promotes both synaptic and intrinsic plasticity of layer II/III pyramidal neurons in the primary motor cortex. Cereb Cortex 26:3494-3507.

Kimpo RR, Doupe AJ (1997) FOS is induced by singing in distinct neuronal populations in a motor network. Neuron 18:315-325.

King AP, West MJ, Goldstein MH (2005) Non-vocal shaping of avian song development: parallels to human speech development. Ethology 111:101-117.

Knudsen El, Knudsen PF (1996) Disruption of auditory spatial working memory by inactivation of the forebrain archistriatum in barn owls. Nature 383:428-431. 
Knudsen El, Cohen YE, Masino T (1995) Characterization of a forebrain gaze field in the archistriatum of the barn owl: microstimulation and anatomical connections. J Neurosci 15:5139-5151.

Kojima S, Doupe AJ (2007) Song selectivity in the pallial-basal ganglia song circuit of zebra finches raised without tutor song exposure. J Neurophysiol 98:2099-2109.

Leonardo A, Fee MS (2005) Ensemble coding of vocal control in birdsong. J Neurosci 25:652-661.

Ljubičić I, Hyland Bruno J, Tchernichovski O (2016) Social influences on song learning. Curr Opin Behav Sci 7:101-107.

Lowndes M, Davies DC (1994) The effects of archistriatal lesions on one-trial passive avoidance learning in the chick. Eur $\mathrm{J}$ Neurosci 6:525-530.

Lowndes M, Davies DC, Johnson MH (1994) Archistriatal lesions impair the acquisition of filial preferences during imprinting in the domestic chick. Eur J Neurosci 6:1143-1148.

Ludwig KA, Miriani RM, Langhals NB, Joseph MD, Anderson DJ, Kipke DR (2009) Using a common average reference to improve cortical neuron recordings from microelectrode arrays. J Neurophysiol 101:16791689.

Makino H, Hwang EJ, Hedrick NG, Komiyama T (2016) Circuit mechanisms of sensorimotor learning. Neuron 92:705-721.

Mandelblat-Cerf Y, Las L, Denisenko N, Fee MS (2014) A role for descending auditory cortical projections in songbird vocal learning. Elife 3:e02152.

Mann NI, Slater PJB (1995) Song tutor choice by zebra finches in aviaries. Anim Behav 49:811-820.

Mann NI, Slater PJB, Eales LA, Richards C (1991) The influence of visual stimuli on song tutor choice in the zebra finch, Taeniopygia guttata. Anim Behav 42:285-293.

Markram H, Toledo-Rodriguez M, Wang Y, Gupta A, Silberberg G, Wu C (2004) Interneurons of the neocortical inhibitory system. Nat Rev Neurosci 5:793-807.

Mello CV, Vates GE, Okuhata S, Nottebohm F (1998) Descending auditory pathways in the adult male zebra finch (Taeniopygia guttata). J Comp Neurol 395:137-160.

Mello CV, Kaser T, Buckner AA, Wirthlin M, Lovell PV (2019) Molecular architecture of the zebra finch arcopallium. J Comp Neurol 527:2512-2556.

Miller-Sims VC, Bottjer SW (2012) Auditory experience refines cortico-basal ganglia inputs to motor cortex via remapping of single axons during vocal learning in zebra finches. J Neurophysiol 107:1142-1156.

Mimica B, Dunn BA, Tombaz T, Srikanth Bojja V, Whitlock JR (2018) Efficient cortical coding of $3 \mathrm{D}$ posture in freely behaving rats. Science 362:584-589.

Moran DW, Schwartz AB (1999) Motor cortical representation of speed and direction during reaching. J Neurophysiol 82:26762692.

Morris D (1954) The reproductive behaviour of the zebra finch (Poephila guttata), with special reference to pseudofemale behaviour and displacement activities. Behaviour 6:271-322.

Morrison RG, Nottebohm F (1993) Role of a telencephalic nucleus in the delayed song learning of socially isolated zebra finches. J Neurobiol 24:1045-1064.

Murata A, Fadiga L, Fogassi L, Gallese V, Raos V, Rizzolatti G (1997) Object representation in the ventral premotor cortex (area F5) of the monkey. J Neurophysiol 78:2226-2230.

Nottebohm F, Stokes TM, Leonard CM (1976) Central control of song in the canary, Serinus canarius. J Comp Neurol 165:457-486.

Ohms VR, Snelderwaard PC, ten Cate C, Beckers GJL (2010) Vocal tract articulation in zebra finches. PLoS One 5:e11923.

Ota N, Gahr M, Soma M (2015) Tap dancing birds: the multimodal mutual courtship display of males and females in a socially monogamous songbird. Sci Rep 5:16614.

Paninski L, Fellows MR, Hatsopoulos NG, Donoghue JP (2004) Spatiotemporal tuning of motor cortical neurons for hand position and velocity. J Neurophysiol 91:515-532.

Papale AE, Hooks BM (2018) Circuit changes in motor cortex during motor skill learning. Neuroscience 368:283-297.
Paterson AK, Bottjer SW (2017) Cortical inter-hemispheric circuits for multimodal vocal learning in songbirds. J Comp Neurol 525:3312-3340.

Peters AJ, Liu H, Komiyama T (2017) Learning in the rodent motor cortex. Annu Rev Neurosci 40:77-97.

Price PH (1979) Developmental determinants of structure in zebra finch song. J Comp Physiol Psychol 93:260-277.

Rauske PL, Shea SD, Margoliash D (2003) State and neuronal classdependent reconfiguration in the avian song system. J Neurophysiol 89:1688-1701.

Reina GA, Moran DW, Schwartz AB (2001) On the relationship between joint angular velocity and motor cortical discharge during reaching. J Neurophysiol 85:2576-2589.

Reiner A, Perkel DJ, Bruce LL, Butler AB, Csillag A, Kuenzel W, Medina L, Paxinos G, Shimizu T, Striedter G, Wild M, Ball GF, Durand S, Güntürkün O, Lee DW, Mello CV, Powers A, White SA, Hough G, Kubikova L, et al. (2004) Revised nomenclature for avian telencephalon and some related brainstem nuclei. J Comp Neurol 473:377-414.

Riede T, Schilling N, Goller F (2013) The acoustic effect of vocal tract adjustments in zebra finches. J Comp Physiol A Neuroethol Sens Neural Behav Physiol 199:57-69.

Rizzolatti G, Luppino G (2001) The cortical motor system. Neuron 31:889-901.

Roper A, Zann R (2006) The onset of song learning and song tutor selection in fledgling zebra finches. Ethology 112:458-470.

Saint-Dizier H, Constantin P, Davies DC, Leterrier C, Lévy F, Richard $S$ (2009) Subdivisions of the arcopallium/posterior pallial amygdala complex are differentially involved in the control of fear behaviour in the Japanese quail. Brain Res Bull 79:288-295.

Schneider C, Devanne H, Lavoie BA, Capaday C (2002) Neural mechanisms involved in the functional linking of motor cortical points. Exp Brain Res 146:86-94.

Schwartz AB, Moran DW (2000) Arm trajectory and representation of movement processing in motor cortical activity. Eur $\mathrm{J}$ Neurosci 12:1851-1856.

Schwartz AB, Kettner RE, Georgopoulos AP (1988) Primate motor cortex and free arm movements to visual targets in three-dimensional space. I. Relations between single cell discharge and direction of movement. J Neurosci 8:2913-2927.

Shen L, Alexander GE (1997) Neural correlates of a spatial sensoryto-motor transformation in primary motor cortex. J Neurophysiol 77:1171-1194.

Sober SJ, Wohlgemuth MJ, Brainard MS (2008) Central contributions to acoustic variation in birdsong. J Neurosci 28:10370-10379.

Spiro J, Dalva M, Mooney R (1999) Long-range inhibition within the zebra finch song nucleus RA can coordinate the firing of multiple projection neurons. J Neurophysiol 81:3007-3020.

Stagg CJ, Bachtiar V, Johansen-Berg H (2011) The role of GABA in human motor learning. Curr Biol 21:480-484.

Steeves JD, Sholomenko GN, Webster DMS (1987) Stimulation of the pontomedullary reticular formation initiates locomotion in decerebrate birds. Brain Res 401:205-212.

Tanji J, Evarts EV (1976) Anticipatory activity of motor cortex neurons in relation to direction of an intended movement. J Neurophysiol 39:1062-1068.

Tomaszycki ML, Adkins-Regan E (2005) Experimental alteration of male song quality and output affects female mate choice and pair bond formation in zebra finches. Anim Behav 70:785-794.

Ullrich R, Norton P, Scharff C (2016) Waltzing Taeniopygia: integration of courtship song and dance in the domesticated Australian zebra finch. Anim Behav 112:285-300.

Valenzuela JI, Hasan SJ, Steeves JD (1990) Stimulation of the brainstem reticular formation evokes locomotor activity in embryonic chicken (in ovo). Brain Res Dev Brain Res 56:13-18.

Vates GE, Broome BM, Mello CV, Nottebohm F (1996) Auditory pathways of caudal telencephalon and their relation to the song system of adult male zebra finches (Taenopygia guttata). J Comp Neurol 366:613-642. 
Vates GE, Vicario DS, Nottebohm F (1997) Reafferent thalamo-"cortical" loops in the song system of oscine songbirds. J Comp Neurol 380:275-290.

Vicario DS, Yohay KH (1993) Song-selective auditory input to a forebrain vocal control nucleus in the zebra finch. J Neurobiol 24:488505.

West MJ, King AP (1988) Female visual displays affect the development of male song in the cowbird. Nature 334:244-246.

Whishaw IQ (2000) Loss of the innate cortical engram for action patterns used in skilled reaching and the development of behavioral compensation following motor cortex lesions in the rat. Neuropharmacology 39:788-805.

Whishaw IQ, Pellis SM, Gorny BP, Pellis VC (1991) The impairments in reaching and the movements of compensation in rats with motor cortex lesions: an endpoint, videorecording, and movement notation analysis. Behav Brain Res 42:77-91.

Wilcox RR, Serang S (2017) Hypothesis testing, p values, confidence intervals, measures of effect size, and Bayesian methods in light of modern robust techniques. Educ Psychol Meas 77:673-689.

Wild JM (1993) Descending projections of the songbird nucleus robustus archistriatalis. J Comp Neurol 338:225-241.
Wild JM, Krützfeldt NEO (2012) Trigeminal and telencephalic projections to jaw and other upper vocal tract premotor neurons in songbirds: sensorimotor circuitry for beak movements during singing. $J$ Comp Neurol 520:590-605.

Williams H (2001) Choreography of song, dance and beak movements in the zebra finch (Taeniopygia guttata). J Exp Biol 204:3497-3506.

Winkowski DE, Knudsen El (2007) Top-down control of multimodal sensitivity in the barn owl optic tectum. J Neurosci 27:1327913291.

Yu AC, Margoliash D (1996) Temporal hierarchical control of singing in birds. Science 273:1871-1875.

Yuan RC, Bottjer SW (2019) Differential developmental changes in cortical representations of auditory-vocal stimuli in songbirds. $J$ Neurophysiol 121:530-548.

Zeier H, Karten HJ (1971) The archistriatum of the pigeon: organization of afferent and efferent connections. Brain Res 31:313-326.

Zhang Q, Yao J, Guang Y, Liang S, Guan J, Qin H, Liao X, Jin W, Zhang J, Pan J, Jia H, Yan J, Feng Z, Li W, Chen X (2017) Locomotion-related population cortical $\mathrm{Ca}+$ transients in freely behaving mice. Front Neural Circuits 11:24. 